\title{
A General Theory of Phase Noise in Electrical Oscillators
}

\author{
Ali Hajimiri, Student Member, IEEE, and Thomas H. Lee, Member, IEEE
}

\begin{abstract}
A general model is introduced which is capable of making accurate, quantitative predictions about the phase noise of different types of electrical oscillators by acknowledging the true periodically time-varying nature of all oscillators. This new approach also elucidates several previously unknown design criteria for reducing close-in phase noise by identifying the mechanisms by which intrinsic device noise and external noise sources contribute to the total phase noise. In particular, it explains the details of how $1 / f$ noise in a device upconverts into close-in phase noise and identifies methods to suppress this upconversion. The theory also naturally accommodates cyclostationary noise sources, leading to additional important design insights. The model reduces to previously available phase noise models as special cases. Excellent agreement among theory, simulations, and measurements is observed.
\end{abstract}

Index Terms-Jitter, oscillator noise, oscillators, oscillator stability, phase jitter, phase locked loops, phase noise, voltage controlled oscillators.

\section{INTRODUCTION}

$\mathbf{T}$ HE recent exponential growth in wireless communication has increased the demand for more available channels in mobile communication applications. In turn, this demand has imposed more stringent requirements on the phase noise of local oscillators. Even in the digital world, phase noise in the guise of jitter is important. Clock jitter directly affects timing margins and hence limits system performance.

Phase and frequency fluctuations have therefore been the subject of numerous studies [1]-[9]. Although many models have been developed for different types of oscillators, each of these models makes restrictive assumptions applicable only to a limited class of oscillators. Most of these models are based on a linear time invariant (LTI) system assumption and suffer from not considering the complete mechanism by which electrical noise sources, such as device noise, become phase noise. In particular, they take an empirical approach in describing the upconversion of low frequency noise sources, such as $1 / f$ noise, into close-in phase noise. These models are also reduced-order models and are therefore incapable of making accurate predictions about phase noise in long ring oscillators, or in oscillators that contain essential singularities, such as delay elements.

Manuscript received December 17, 1996; revised July 9, 1997.

The authors are with the Center for Integrated Systems, Stanford University, Stanford, CA 94305-4070 USA.

Publisher Item Identifier S 0018-9200(98)00716-1.
Since any oscillator is a periodically time-varying system, its time-varying nature must be taken into account to permit accurate modeling of phase noise. Unlike models that assume linearity and time-invariance, the time-variant model presented here is capable of proper assessment of the effects on phase noise of both stationary and even of cyclostationary noise sources.

Noise sources in the circuit can be divided into two groups, namely, device noise and interference. Thermal, shot, and flicker noise are examples of the former, while substrate and supply noise are in the latter group. This model explains the exact mechanism by which spurious sources, random or deterministic, are converted into phase and amplitude variations, and includes previous models as special limiting cases.

This time-variant model makes explicit predictions of the relationship between waveform shape and $1 / f$ noise upconversion. Contrary to widely held beliefs, it will be shown that the $1 / f^{3}$ corner in the phase noise spectrum is smaller than $1 / f$ noise corner of the oscillator's components by a factor determined by the symmetry properties of the waveform. This result is particularly important in CMOS RF applications because it shows that the effect of inferior $1 / f$ device noise can be reduced by proper design.

Section II is a brief introduction to some of the existing phase noise models. Section III introduces the time-variant model through an impulse response approach for the excess phase of an oscillator. It also shows the mechanism by which noise at different frequencies can become phase noise and expresses with a simple relation the sideband power due to an arbitrary source (random or deterministic). It continues with explaining how this approach naturally lends itself to the analysis of cyclostationary noise sources. It also introduces a general method to calculate the total phase noise of an oscillator with multiple nodes and multiple noise sources, and how this method can help designers to spot the dominant source of phase noise degradation in the circuit. It concludes with a demonstration of how the presented model reduces to existing models as special cases. Section IV gives new design implications arising from this theory in the form of guidelines for low phase noise design. Section V concludes with experimental results supporting the theory.

\section{BRIEF REVIEW of Existing Models AND DEFinitions}

The output of an ideal sinusoidal oscillator may be expressed as $V_{\text {out }}(t)=A \cos \left[\omega_{0} t+\phi\right]$, where $A$ is the amplitude, 


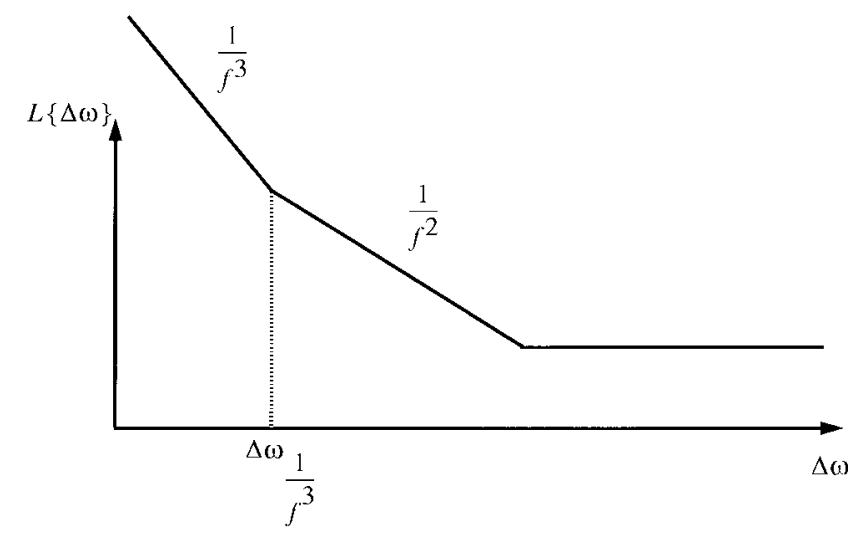

Fig. 1. Typical plot of the phase noise of an oscillator versus offset from carrier.

$\omega_{0}$ is the frequency, and $\phi$ is an arbitrary, fixed phase reference. Therefore, the spectrum of an ideal oscillator with no random fluctuations is a pair of impulses at $\pm \omega_{0}$. In a practical oscillator, however, the output is more generally given by

$$
V_{\text {out }}(t)=A(t) \cdot f\left[\omega_{0} t+\phi(t)\right]
$$

where $\phi(t)$ and $A(t)$ are now functions of time and $f$ is a periodic function with period $2 \pi$. As a consequence of the fluctuations represented by $\phi(t)$ and $A(t)$, the spectrum of a practical oscillator has sidebands close to the frequency of oscillation, $\omega_{0}$.

There are many ways of quantifying these fluctuations (a comprehensive review of different standards and measurement methods is given in [4]). A signal's short-term instabilities are usually characterized in terms of the single sideband noise spectral density. It has units of decibels below the carrier per hertz $(\mathrm{dBc} / \mathrm{Hz})$ and is defined as

$$
\mathcal{L}_{\text {total }}\{\Delta \omega\}=10 \cdot \log \left[\frac{\mathcal{P}_{\text {sideband }}\left(\omega_{0}+\Delta \omega, 1 \mathrm{~Hz}\right)}{\mathcal{P}_{\text {carrier }}}\right]
$$

where $P_{\text {sideband }}\left(\omega_{0}+\Delta \omega, 1 \mathrm{~Hz}\right)$ represents the single sideband power at a frequency offset of $\Delta \omega$ from the carrier with a measurement bandwidth of $1 \mathrm{~Hz}$. Note that the above definition includes the effect of both amplitude and phase fluctuations, $A(t)$ and $\phi(t)$.

The advantage of this parameter is its ease of measurement. Its disadvantage is that it shows the sum of both amplitude and phase variations; it does not show them separately. However, it is important to know the amplitude and phase noise separately because they behave differently in the circuit. For instance, the effect of amplitude noise is reduced by amplitude limiting mechanism and can be practically eliminated by the application of a limiter to the output signal, while the phase noise cannot be reduced in the same manner. Therefore, in most applications, $\mathcal{L}_{\text {total }}\{\Delta \omega\}$ is dominated by its phase portion, $\mathcal{L}_{\text {phase }}\{\Delta \omega\}$, known as phase noise, which we will simply denote as $\mathcal{L}\{\Delta \omega\}$.

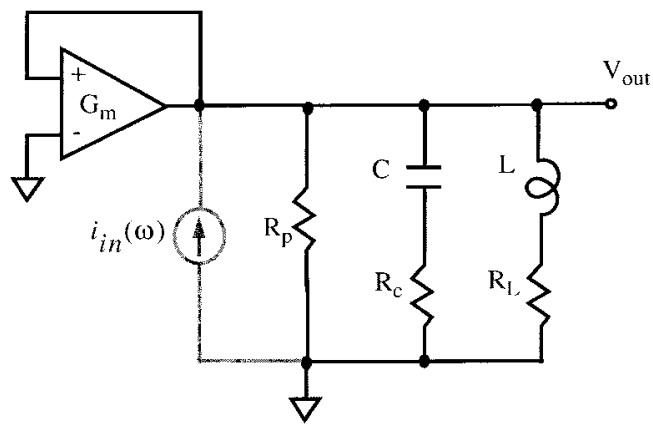

Fig. 2. A typical RLC oscillator.

The semi-empirical model proposed in [1]-[3], known also as the Leeson-Cutler phase noise model, is based on an LTI assumption for tuned tank oscillators. It predicts the following behavior for $\mathcal{L}\{\Delta \omega\}$ :

$$
\begin{gathered}
\mathcal{L}\{\Delta \omega\}=10 \cdot \log \left\{\frac{2 F k T}{P_{s}} \cdot\left[1+\left(\frac{\omega_{0}}{2 Q_{L} \Delta \omega}\right)^{2}\right]\right. \\
\left.\cdot\left(1+\frac{\Delta \omega_{1 / f^{3}}}{|\Delta \omega|}\right)\right\}
\end{gathered}
$$

where $F$ is an empirical parameter (often called the "device excess noise number"), $k$ is Boltzmann's constant, $T$ is the absolute temperature, $P_{s}$ is the average power dissipated in the resistive part of the tank, $\omega_{0}$ is the oscillation frequency, $Q_{L}$ is the effective quality factor of the tank with all the loadings in place (also known as loaded $Q$ ), $\Delta \omega$ is the offset from the carrier and $\Delta \omega_{1 / f}^{3}$ is the frequency of the corner between the $1 / f^{3}$ and $1 / f^{2}$ regions, as shown in the sideband spectrum of Fig. 1 . The behavior in the $1 / f^{2}$ region can be obtained by applying a transfer function approach as follows. The impedance of a parallel RLC, for $\Delta \omega \ll \omega_{0}$, is easily calculated to be

$$
Z\left(\omega_{0}+\Delta \omega\right) \approx \frac{1}{G_{L}} \cdot \frac{1}{1+j 2 Q_{L} \frac{\Delta \omega}{\omega_{0}}}
$$

where $G_{L}$ is the parallel parasitic conductance of the tank. For steady-state oscillation, the equation $G_{m} R_{L}=1$ should be satisfied. Therefore, for a parallel current source, the closedloop transfer function of the oscillator shown in Fig. 2 is given by the imaginary part of the impedance

$$
H(\Delta \omega)=\frac{v_{\text {out }}\left(\omega_{0}+\Delta \omega\right)}{i_{\text {in }}\left(\omega_{0}+\Delta \omega\right)}=-j \frac{1}{G_{L}} \cdot \frac{\omega_{0}}{2 Q_{L} \Delta \omega} .
$$

The total equivalent parallel resistance of the tank has an equivalent mean square noise current density of $\overline{i_{n}^{2}} / \Delta f=$ $4 k T G_{L}$. In addition, active device noise usually contributes a significant portion of the total noise in the oscillator. It is traditional to combine all the noise sources into one effective noise source, expressed in terms of the resistor noise with 


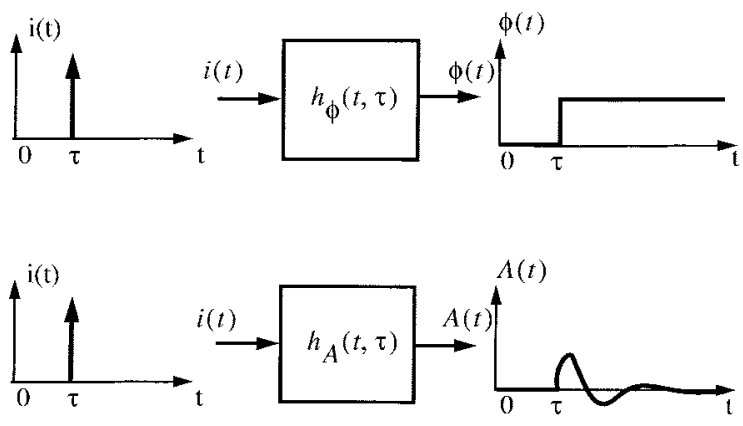

Fig. 3. Phase and amplitude impulse response model.

a multiplicative factor, $F$, known as the device excess noise number. The equivalent mean square noise current density can therefore be expressed as $\overline{i_{n}^{2}} / \Delta f=4 F k T G_{L}$. Unfortunately, it is generally difficult to calculate $F$ a priori. One important reason is that much of the noise in a practical oscillator arises from periodically varying processes and is therefore cyclostationary. Hence, as mentioned in [3], $F$ and $\omega_{1 / f}^{3}$ are usually used as a posteriori fitting parameters on measured data.

Using the above effective noise current power, the phase noise in the $1 / f^{2}$ region of the spectrum can be calculated as

$$
\begin{aligned}
\mathcal{L}\{\Delta \omega\} & =10 \cdot \log \left(\frac{\overline{v_{\text {noise }}^{2}}}{\overline{v_{\text {sig }}^{2}}}\right) \\
& =10 \cdot \log \left[\frac{\frac{1}{2} \cdot|H(\Delta \omega)|^{2} \cdot \overline{i_{n}^{2}} / \Delta f}{\frac{1}{2} \cdot V_{\max }^{2}}\right] \\
& =10 \cdot \log \left[\frac{2 F k T}{P_{s}} \cdot\left(\frac{\omega_{0}}{2 Q \Delta \omega}\right)^{2}\right] .
\end{aligned}
$$

Note that the factor of $1 / 2$ arises from neglecting the contribution of amplitude noise. Although the expression for the noise in the $1 / f^{2}$ region is thus easily obtained, the expression for the $1 / f^{3}$ portion of the phase noise is completely empirical. As such, the common assumption that the $1 / f^{3}$ corner of the phase noise is the same as the $1 / f$ corner of device flicker noise has no theoretical basis.

The above approach may be extended by identifying the individual noise sources in the tuned tank oscillator of Fig. 2 [8]. An LTI approach is used and there is an embedded assumption of no amplitude limiting, contrary to most practical cases. For the RLC circuit of Fig. 2, [8] predicts the following:

$$
\mathcal{L}\{\Delta \omega\}=10 \cdot \log \left[\frac{k \mathcal{T} \cdot \mathcal{R}_{\text {eff }}[1+\mathcal{A}] \cdot\left(\frac{\omega_{n}}{\Delta \omega}\right)^{2}}{\mathcal{V}_{\max }^{2} / 2}\right]
$$

where $A$ is yet another empirical fitting parameter, and $R_{\mathrm{eff}}$ is the effective series resistance, given by

$$
R_{\mathrm{eff}}=R_{L}+R_{C}+\frac{1}{R_{p}\left(C \omega_{0}\right)^{2}}
$$

where $R_{L}, R_{C}, R_{p}$, and $C$ are shown in Fig. 2. Note that it is still not clear how to calculate $A$ from circuit parameters. Hence, this approach represents no fundamental improvement over the method outlined in [3].

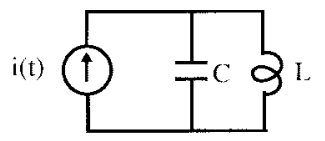

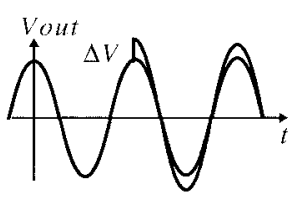

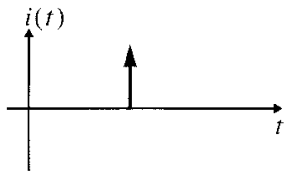

(a)
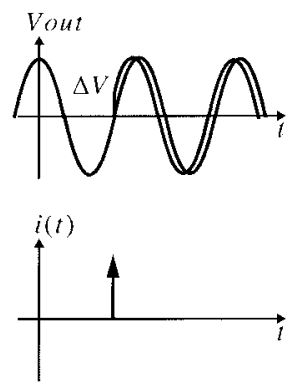

(b)

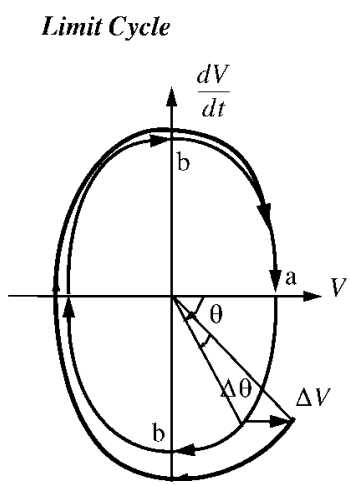

(c)

Fig. 4. (a) Impulse injected at the peak, (b) impulse injected at the zero crossing, and (c) effect of nonlinearity on amplitude and phase of the oscillator in state-space.

\section{Modeling OF PhASE NoISE}

\section{A. Impulse Response Model for Excess Phase}

An oscillator can be modeled as a system with $n$ inputs (each associated with one noise source) and two outputs that are the instantaneous amplitude and excess phase of the oscillator, $A(t)$ and $\phi(t)$, as defined by (1). Noise inputs to this system are in the form of current sources injecting into circuit nodes and voltage sources in series with circuit branches. For each input source, both systems can be viewed as singleinput, single-output systems. The time and frequency-domain fluctuations of $A(t)$ and $\phi(t)$ can be studied by characterizing the behavior of two equivalent systems shown in Fig. 3 .

Note that both systems shown in Fig. 3 are time variant. Consider the specific example of an ideal parallel $L C$ oscillator shown in Fig. 4. If we inject a current impulse $i(t)$ as shown, the amplitude and phase of the oscillator will have responses similar to that shown in Fig. 4(a) and (b). The instantaneous voltage change $\Delta V$ is given by

$$
\Delta V=\frac{\Delta q}{C_{\text {tot }}}
$$

where $\Delta q$ is the total injected charge due to the current impulse and $C_{\text {tot }}$ is the total capacitance at that node. Note that the current impulse will change only the voltage across the 


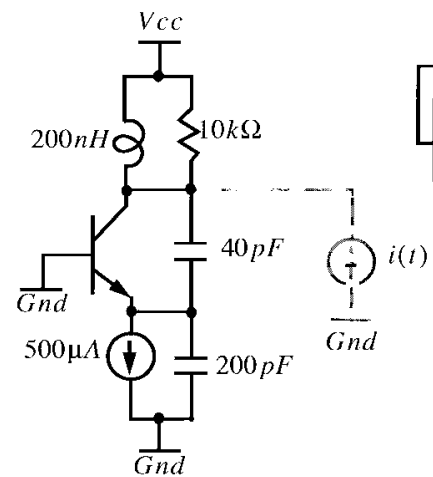

(a)

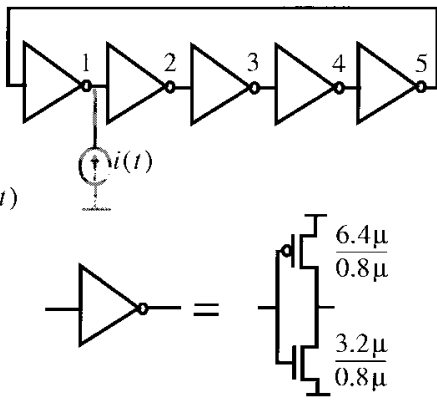

(b)
Fig. 5. (a) A typical Colpitts oscillator and (b) a five-stage minimum size ring oscillator.

capacitor and will not affect the current through the inductor. It can be seen from Fig. 4 that the resultant change in $A(t)$ and $\phi(t)$ is time dependent. In particular, if the impulse is applied at the peak of the voltage across the capacitor, there will be no phase shift and only an amplitude change will result, as shown in Fig. 4(a). On the other hand, if this impulse is applied at the zero crossing, it has the maximum effect on the excess phase $\phi(t)$ and the minimum effect on the amplitude, as depicted in Fig. 4(b). This time dependence can also be observed in the state-space trajectory shown in Fig. 4(c). Applying an impulse at the peak is equivalent to a sudden jump in voltage at point $a$, which results in no phase change and changes only the amplitude, while applying an impulse at point $\boldsymbol{b}$ results only in a phase change without affecting the amplitude. An impulse applied sometime between these two extremes will result in both amplitude and phase changes.

There is an important difference between the phase and amplitude responses of any real oscillator, because some form of amplitude limiting mechanism is essential for stable oscillatory action. The effect of this limiting mechanism is pictured as a closed trajectory in the state-space portrait of the oscillator shown in Fig. 4(c). The system state will finally approach this trajectory, called a limit cycle, irrespective of its starting point [10]-[12]. Both an explicit automatic gain control (AGC) and the intrinsic nonlinearity of the devices act similarly to produce a stable limit cycle. However, any fluctuation in the phase of the oscillation persists indefinitely, with a current noise impulse resulting in a step change in phase, as shown in Fig. 3. It is important to note that regardless of how small the injected charge, the oscillator remains time variant.

Having established the essential time-variant nature of the systems of Fig. 3, we now show that they may be treated as linear for all practical purposes, so that their impulse responses $h_{\phi}(t, \tau)$ and $h_{A}(t, \tau)$ will characterize them completely.

The linearity assumption can be verified by injecting impulses with different areas (charges) and measuring the resultant phase change. This is done in the SPICE simulations of the 62-MHz Colpitts oscillator shown in Fig. 5(a) and the fivestage 1.01-GHz, 0.8- $\mu \mathrm{m}$ CMOS inverter chain ring oscillator shown in Fig. 5(b). The results are shown in Fig. 6(a) and (b), respectively. The impulse is applied close to a zero crossing,

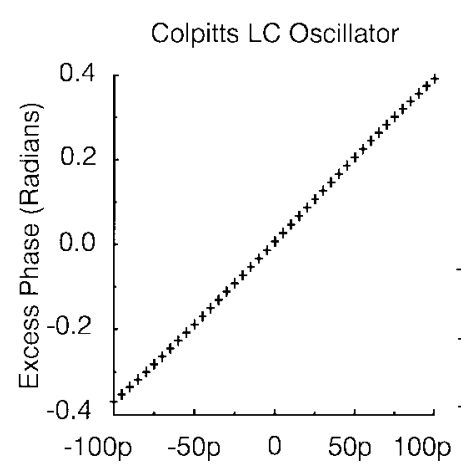

Injected Charge (coulombs)

(a)
Five Stage Ring Oscillator

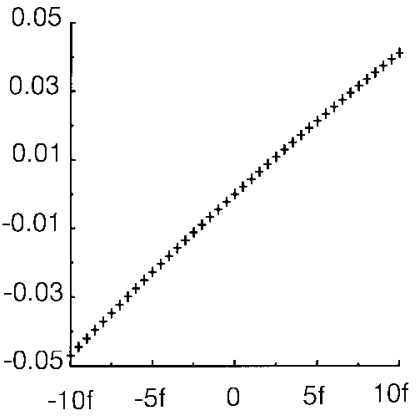

Injected Charge (coulombs)

(b)
Fig. 6. Phase shift versus injected charge for oscillators of Fig. 5(a) and (b).

where it has the maximum effect on phase. As can be seen, the current-phase relation is linear for values of charge up to $10 \%$ of the total charge on the effective capacitance of the node of interest. Also note that the effective injected charges due to actual noise and interference sources in practical circuits are several orders of magnitude smaller than the amounts of charge injected in Fig. 6. Thus, the assumption of linearity is well satisfied in all practical oscillators.

It is critical to note that the current-to-phase transfer function is practically linear even though the active elements may have strongly nonlinear voltage-current behavior. However, the nonlinearity of the circuit elements defines the shape of the limit cycle and has an important influence on phase noise that will be accounted for shortly.

We have thus far demonstrated linearity, with the amount of excess phase proportional to the ratio of the injected charge to the maximum charge swing across the capacitor on the node, i.e., $\Delta q / q_{\max }$. Furthermore, as discussed earlier, the impulse response for the first system of Fig. 3 is a step whose amplitude depends periodically on the time $\tau$ when the impulse is injected. Therefore, the unit impulse response for excess phase can be expressed as

$$
h_{\phi}(t, \tau)=\frac{\Gamma\left(\omega_{0} \tau\right)}{q_{\max }} u(t-\tau)
$$

where $q_{\max }$ is the maximum charge displacement across the capacitor on the node and $u(t)$ is the unit step. We call $\Gamma(x)$ the impulse sensitivity function (ISF). It is a dimensionless, frequency- and amplitude-independent periodic function with period $2 \pi$ which describes how much phase shift results from applying a unit impulse at time $t=\tau$. To illustrate its significance, the ISF's together with the oscillation waveforms for a typical $L C$ and ring oscillator are shown in Fig. 7. As is shown in the Appendix, $\Gamma(x)$ is a function of the waveform or, equivalently, the shape of the limit cycle which, in turn, is governed by the nonlinearity and the topology of the oscillator.

Given the ISF, the output excess phase $\phi(t)$ can be calculated using the superposition integral

$$
\phi(t)=\int_{-\infty}^{\infty} h_{\phi}(t, \tau) i(\tau) d \tau=\frac{1}{q_{\max }} \int_{-\infty}^{t} \Gamma\left(\omega_{0} \tau\right) i(\tau) d \tau
$$




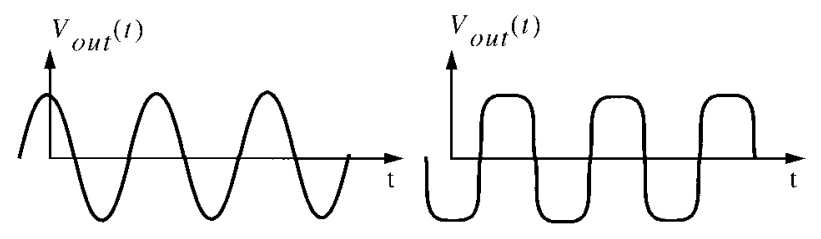

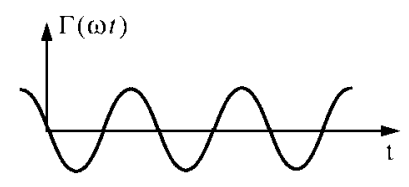

(a)

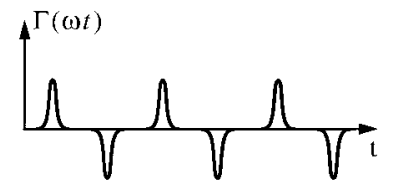

(b)
Fig. 7. Waveforms and ISF's for (a) a typical $L C$ oscillator and (b) a typical ring oscillator.

where $i(t)$ represents the input noise current injected into the node of interest. Since the ISF is periodic, it can be expanded in a Fourier series

$$
\Gamma\left(\omega_{0} \tau\right)=\frac{c_{0}}{2}+\sum_{n=1}^{\infty} c_{n} \cos \left(n \omega_{0} \tau+\theta_{n}\right)
$$

where the coefficients $c_{n}$ are real-valued coefficients, and $\theta_{n}$ is the phase of the $n$th harmonic. As will be seen later, $\theta_{n}$ is not important for random input noise and is thus neglected here. Using the above expansion for $\Gamma\left(\omega_{0} \tau\right)$ in the superposition integral, and exchanging the order of summation and integration, we obtain

$$
\begin{aligned}
\phi(t)=\frac{1}{q_{\max }}\left[\frac{c_{0}}{2} \int_{-\infty}^{t} i(\tau) d \tau\right. \\
\left.+\sum_{n=1}^{\infty} c_{n} \int_{-\infty}^{t} i(\tau) \cos \left(n \omega_{0} \tau\right) d \tau\right]
\end{aligned}
$$

Equation (13) allows computation of $\phi(t)$ for an arbitrary input current $i(t)$ injected into any circuit node, once the various Fourier coefficients of the ISF have been found.

As an illustrative special case, suppose that we inject a low frequency sinusoidal perturbation current $i(t)$ into the node of interest at a frequency of $\Delta \omega \ll \omega_{0}$

$$
i(t)=I_{0} \cos (\Delta \omega t)
$$

where $I_{0}$ is the maximum amplitude of $i(t)$. The arguments of all the integrals in (13) are at frequencies higher than $\omega_{0}$ and are significantly attenuated by the averaging nature of the integration, except the term arising from the first integral, which involves $c_{0}$. Therefore, the only significant term in $\phi(t)$ will be

$$
\phi(t) \approx \frac{I_{0} c_{0}}{2 q_{\max }} \int_{-\infty}^{t} \cos (\Delta \omega \tau) d \tau=\frac{I_{0} c_{0} \sin (\Delta \omega t)}{2 q_{\max } \Delta \omega} .
$$

As a result, there will be two impulses at $\pm \Delta \omega$ in the power spectral density of $\phi(t)$, denoted as $S_{\phi}(\omega)$.

As an important second special case, consider a current at a frequency close to the carrier injected into the node of interest, given by $i(t)=I_{1} \cos \left[\left(\omega_{0}+\Delta \omega\right) t\right]$. A process similar to that of the previous case occurs except that the spectrum of $i(t)$

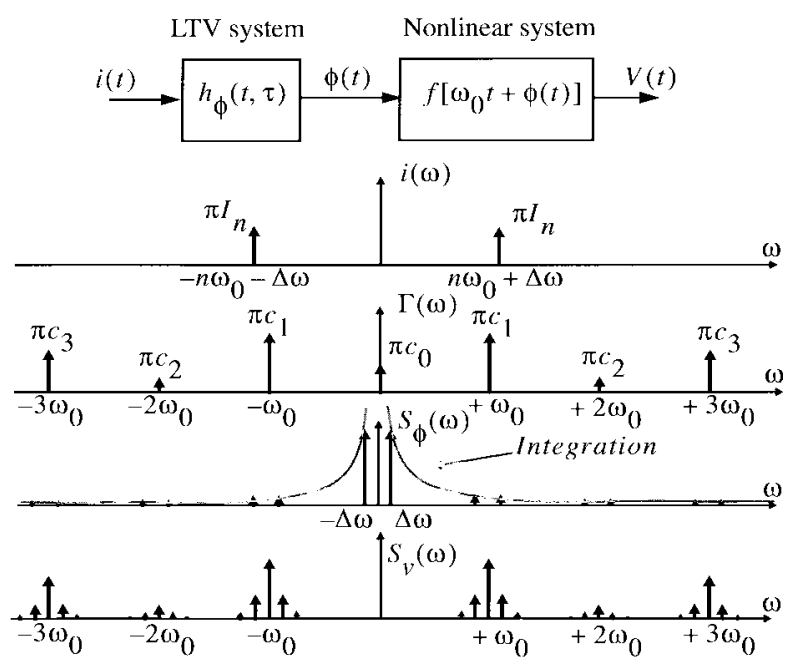

Fig. 8. Conversion of the noise around integer multiples of the oscillation frequency into phase noise.

consists of two impulses at $\pm\left(\omega_{0}+\Delta \omega\right)$ as shown in Fig. 8 . This time the only integral in (13) which will have a low frequency argument is for $n=1$. Therefore $\phi(t)$ is given by

$$
\phi(t) \approx \frac{I_{1} c_{1} \sin (\Delta \omega t)}{2 q_{\max } \Delta \omega}
$$

which again results in two equal sidebands at $\pm \Delta \omega$ in $S_{\phi}(\omega)$.

More generally, (13) suggests that applying a current $i(t)=$ $I_{n} \cos \left[\left(n \omega_{0}+\Delta \omega\right) t\right]$ close to any integer multiple of the oscillation frequency will result in two equal sidebands at $\pm \Delta \omega$ in $S_{\phi}(\omega)$. Hence, in the general case $\phi(t)$ is given by

$$
\phi(t) \approx \frac{I_{n} c_{n} \sin (\Delta \omega t)}{2 q_{\max } \Delta \omega} .
$$

\section{B. Phase-to-Voltage Transformation}

So far, we have presented a method for determining how much phase error results from a given current $i(t)$ using (13). Computing the power spectral density (PSD) of the oscillator output voltage $S_{v}(\omega)$ requires knowledge of how the output voltage relates to the excess phase variations. As shown in Fig. 8, the conversion of device noise current to output voltage may be treated as the result of a cascade of two processes. The first corresponds to a linear time variant (LTV) currentto-phase converter discussed above, while the second is a nonlinear system that represents a phase modulation (PM), which transforms phase to voltage. To obtain the sideband power around the fundamental frequency, the fundamental harmonic of the oscillator output $\cos \left[\omega_{0} t+\phi(t)\right]$ can be used as the transfer function for the second system in Fig. 8. Note this is a nonlinear transfer function with $\phi(t)$ as the input.

Substituting $\phi(t)$ from (17) into (1) results in a single-tone phase modulation for output voltage, with $\phi(t)$ given by (17). Therefore, an injected current at $n \omega_{0}+\Delta \omega$ results in a pair of equal sidebands at $\omega_{0} \pm \Delta \omega$ with a sideband power relative to the carrier given by

$$
P_{\mathrm{SBC}}(\Delta \omega)=10 \cdot \log \left(\frac{I_{n} c_{n}}{4 q_{\max } \Delta \omega}\right)^{2}
$$


Low Frequency Current Injection Injection Close to $f_{0}$

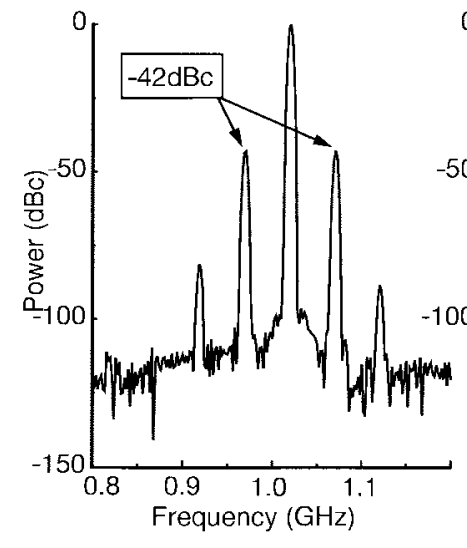

(a)

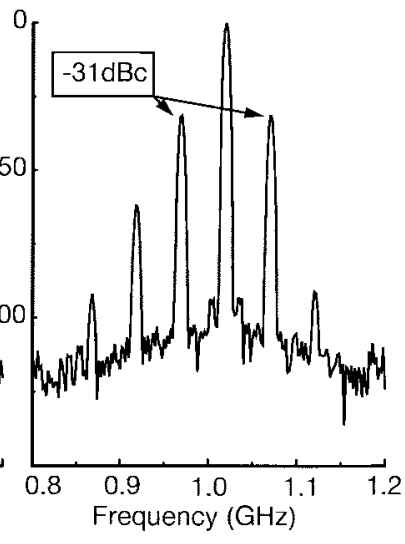

(b)
Fig. 9. Simulated power spectrum of the output with current injection at (a) $f_{m}=50 \mathrm{MHz}$ and (b) $f_{0}+f_{m}=1.06 \mathrm{GHz}$.

This process is shown in Fig. 8. Appearance of the frequency deviation $\Delta \omega$ in the denominator of the (18) underscores that the impulse response $h_{\phi}(t, \tau)$ is a step function and therefore behaves as a time-varying integrator. We will frequently refer to (18) in subsequent sections.

Applying this method of analysis to an arbitrary oscillator, a sinusoidal current injected into one of the oscillator nodes at a frequency $\omega_{0}+\Delta \omega$ results in two equal sidebands at $\omega_{0} \pm \Delta \omega$, as observed in [9]. Note that it is necessary to use an LTV because an LTI model cannot explain the presence of a pair of equal sidebands close to the carrier arising from sources at frequencies $n \omega_{0}+\Delta \omega$, because an LTI system cannot produce any frequencies except those of the input and those associated with the system's poles. Furthermore, the amplitude of the resulting sidebands, as well as their equality, cannot be predicted by conventional intermodulation effects. This failure is to be expected since the intermodulation terms arise from nonlinearity in the voltage (or current) input/output characteristic of active devices of the form $V_{\text {out }}=\alpha_{1} V_{\text {in }}+$ $\alpha_{2} V_{\mathrm{in}}^{2}+\alpha_{3} V_{\mathrm{in}}^{3}+\cdots$. This type of nonlinearity does not directly appear in the phase transfer characteristic and shows itself only indirectly in the ISF.

It is instructive to compare the predictions of (18) with simulation results. A sinusoidal current of $10 \mu \mathrm{A}$ amplitude at different frequencies was injected into node 1 of the $1.01-\mathrm{GHz}$ ring oscillator of Fig. 5(b). Fig. 9(a) shows the simulated power spectrum of the signal on node 4 for a low frequency input at $f_{m}=50 \mathrm{MHz}$. This power spectrum is obtained using the fast Fourier transform (FFT) analysis in HSPICE 96.1. It is noteworthy that in this version of HSPICE the simulation artifacts observed in [9] have been properly eliminated by calculation of the values used in the analysis at the exact points of interest. Note that the injected noise is upconverted into two equal sidebands at $f_{0}+f_{m}$ and $f_{0}-f_{m}$, as predicted by (18). Fig. 9(b) shows the effect of injection of a current at $f_{0}+f_{m}=1.06 \mathrm{GHz}$. Again, two equal sidebands are observed at $f_{0}+f_{m}$ and $f_{0}-f_{m}$, also as predicted by (18).

Simulated sideband power for the general case of current injection at $n f_{0}+f_{m}$ can be compared to the predictions of
5-Stage Ring Oscillator Sideband Power

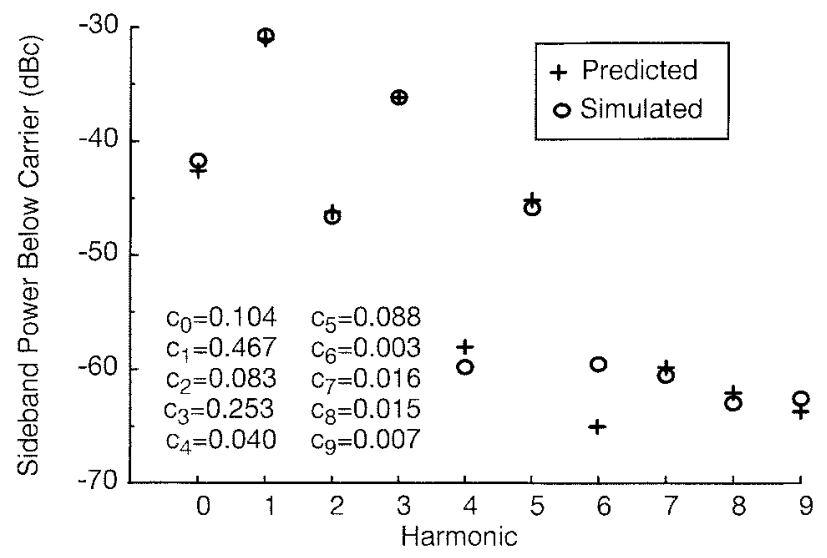

Fig. 10. Simulated and calculated sideband powers for the first ten coefficients.

(18). The ISF for this oscillator is obtained by the simulation method of the Appendix. Here, $q_{\max }$ is equal to $C_{\mathrm{eq}} V_{\text {swing }}$, where $C_{\mathrm{eq}}$ is the average capacitance on each node of the circuit and $V_{\text {swing }}$ is the maximum swing across it. For this oscillator, $C_{\mathrm{eq}}=26 \mathrm{fF}$ and $V_{\text {swing }}=5 \mathrm{~V}$, which results in $q_{\max }=130 \mathrm{fC}$. For a sinusoidal injected current of amplitude $I_{n}=10 \mu \mathrm{A}$, and an $f_{m}$ of $50 \mathrm{MHz}$, Fig. 10 depicts the simulated and predicted sideband powers. As can be seen from the figure, these agree to within $1 \mathrm{~dB}$ for the higher power sidebands. The discrepancy in the case of the low power sidebands $(n=4,6-9)$ arises from numerical noise in the simulations, which represents a greater fractional error at lower sideband power. Overall, there is satisfactory agreement between simulation and the theory of conversion of noise from various frequencies into phase fluctuations.

\section{Prediction of Phase Noise Sideband Power}

Now we consider the case of a random noise current $i_{n}(t)$ whose power spectral density has both a flat region and a $1 / f$ region, as shown in Fig. 11. As can be seen from (18) and the foregoing discussion, noise components located near integer multiples of the oscillation frequency are transformed to low frequency noise sidebands for $S_{\phi}(\omega)$, which in turn become close-in phase noise in the spectrum of $S_{v}(\omega)$, as illustrated in Fig. 11. It can be seen that the total $S_{\phi}(\omega)$ is given by the sum of phase noise contributions from device noise in the vicinity of the integer multiples of $\omega_{0}$, weighted by the coefficients $c_{n}$. This is shown in Fig. 12(a) (logarithmic frequency scale). The resulting single sideband spectral noise density $L\{\Delta \omega\}$ is plotted on a logarithmic scale in Fig. 12(b). The sidebands in the spectrum of $S_{\phi}(\omega)$, in turn, result in phase noise sidebands in the spectrum of $S_{v}(\omega)$ through the PM mechanism discuss in the previous subsection. This process is shown in Figs. 11 and 12.

The theory predicts the existence of $1 / f^{3}, 1 / f^{2}$, and flat regions for the phase noise spectrum. The low-frequency noise sources, such as flicker noise, are weighted by the coefficient $c_{0}$ and show a $1 / f^{3}$ dependence on the offset frequency, while 


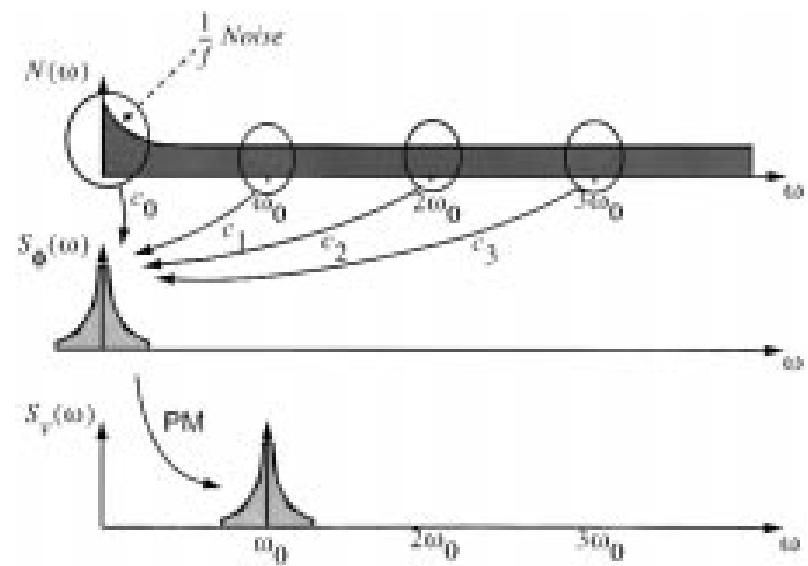

Fig. 11. Conversion of noise to phase fluctuations and phase-noise sidebands.

the white noise terms are weighted by other $c_{n}$ coefficients and give rise to the $1 / f^{2}$ region of phase noise spectrum. It is apparent that if the original noise current $i(t)$ contains $1 / f^{n}$ low frequency noise terms, such as popcorn noise, they can appear in the phase noise spectrum as $1 / f^{n+2}$ regions. Finally, the flat noise floor in Fig. 12(b) arises from the white noise floor of the noise sources in the oscillator. The total sideband noise power is the sum of these two as shown by the bold line in the same figure.

To carry out a quantitative analysis of the phase noise sideband power, now consider an input noise current with a white power spectral density $\overline{i_{n}^{2}} / \Delta f$. Note that $I_{n}$ in (18) represents the peak amplitude, hence, $I_{n}^{2} / 2=\overline{i_{n}^{2}} / \Delta f$ for $\Delta f=1 \mathrm{~Hz}$. Based on the foregoing development and (18), the total single sideband phase noise spectral density in $\mathrm{dB}$ below the carrier per unit bandwidth due to the source on one node at an offset frequency of $\Delta \omega$ is given by

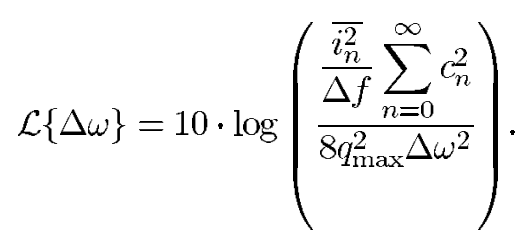

Now, according to Parseval's relation we have

$$
\sum_{n=0}^{\infty} c_{n}^{2}=\frac{1}{\pi} \int_{0}^{2 \pi}|\Gamma(x)|^{2} d x=2 \Gamma_{\mathrm{rms}}^{2}
$$

where $\Gamma_{\mathrm{rms}}$ is the rms value of $\Gamma(x)$. As a result

$$
\mathcal{L}\{\Delta \omega\}=10 \cdot \log \left(\frac{\Gamma_{\mathrm{rms}}^{2}}{q_{\max }^{2}} \cdot \frac{\overline{i_{n}^{2}} / \Delta f}{4 \cdot \Delta \omega^{2}}\right) .
$$

This equation represents the phase noise spectrum of an arbitrary oscillator in $1 / f^{2}$ region of the phase noise spectrum. For a voltage noise source in series with an inductor, $q_{\max }$ should be replaced with $\Phi_{\max }=L I_{\text {swing, }}$, where $\Phi_{\max }$ represents the maximum magnetic flux swing in the inductor.

We may now investigate quantitatively the relationship between the device $1 / f$ corner and the $1 / f^{3}$ corner of the phase noise. It is important to note that it is by no means (a)

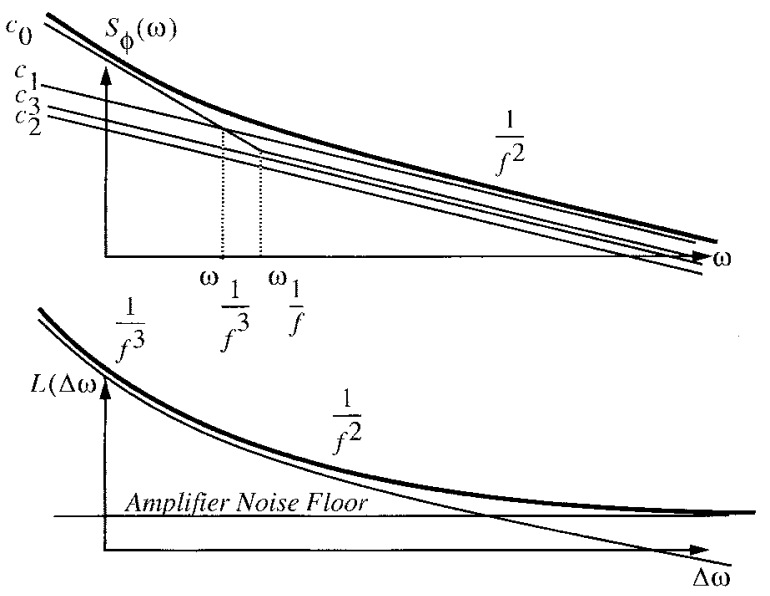

Fig. 12. (a) PSD of $\phi(t)$ and (b) single sideband phase noise power spectrum, $L\{\Delta \omega\}$.

obvious from the foregoing development that the $1 / f^{3}$ corner of the phase noise and the $1 / f$ corner of the device noise should be coincident, as is commonly assumed. In fact, from Fig. 12, it should be apparent that the relationship between these two frequencies depends on the specific values of the various coefficients $c_{n}$. The device noise in the flicker noise dominated portion of the noise spectrum $\left(\Delta \omega<\omega_{1 / f}\right)$ can be described by

$$
{\overline{i^{2}}}_{n, 1 / f}=\overline{i_{n}^{2}} \cdot \frac{\omega_{1 / f}}{\Delta \omega} \quad\left(\Delta \omega<\omega_{1 / f}\right)
$$

where $\omega_{1 / f}$ is the corner frequency of device $1 / f$ noise. Equation (22) together with (18) result in the following expression for phase noise in the $1 / f^{3}$ portion of the phase noise spectrum:

$$
\mathcal{L}\{\Delta \omega\}=10 \cdot \log \left(\frac{c_{0}^{2}}{q_{\max }^{2}} \cdot \frac{\overline{i_{n}^{2}} / \Delta f}{8 \cdot \Delta \omega^{2}} \cdot \frac{\omega_{1 / f}}{\Delta \omega}\right) .
$$

The phase noise $1 / f^{3}$ corner, $\omega_{1 / f^{3}}$, is the frequency where the sideband power due to the white noise given by (21) is equal to the sideband power arising from the $1 / f$ noise given by (23), as shown in Fig. 12. Solving for $\omega_{1 / f^{3}}$ results in the following expression for the $1 / f^{3}$ corner in the phase noise spectrum:

$$
\omega_{1 / f^{3}}=\omega_{1 / f} \cdot \frac{c_{0}^{2}}{2 \Gamma_{\mathrm{rms}}^{2}} \approx \omega_{1 / f} \cdot\left(\frac{c_{0}}{c_{1}}\right)^{2} .
$$

This equation together with (21) describe the phase noise spectrum and are the major results of this section. As can be seen, the $1 / f^{3}$ phase noise corner due to internal noise sources is not equal to the $1 / f$ device noise corner, but is smaller by a factor equal to $c_{0}^{2} / 2 \Gamma_{\mathrm{rms}}$. As will be discussed later, $c_{0}$ depends on the waveform and can be significantly reduced if certain symmetry properties exist in the waveform of the oscillation. Thus, poor $1 / f$ device noise need not imply poor close-in phase noise performance. 


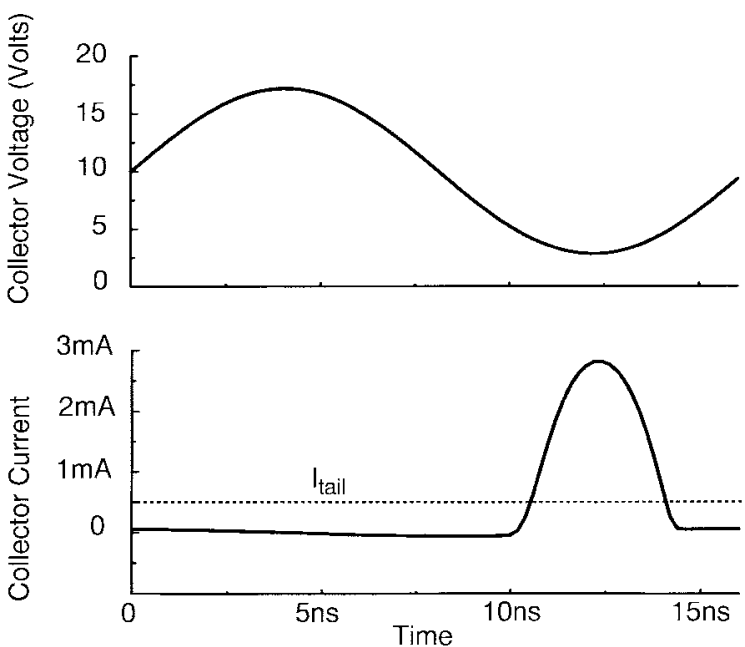

Fig. 13. Collector voltage and collector current of the Colpitts oscillator of Fig. 5(a).

\section{Cyclostationary Noise Sources}

In addition to the periodically time-varying nature of the system itself, another complication is that the statistical properties of some of the random noise sources in the oscillator may change with time in a periodic manner. These sources are referred to as cyclostationary. For instance, the channel noise of a MOS device in an oscillator is cyclostationary because the noise power is modulated by the gate source overdrive which varies with time periodically. There are other noise sources in the circuit whose statistical properties do not depend on time and the operation point of the circuit, and are therefore called stationary. Thermal noise of a resistor is an example of a stationary noise source.

A white cyclostationary noise current $i_{n}(t)$ can be decomposed as [13]:

$$
i_{n}(t)=i_{n 0}(t) \cdot \alpha\left(\omega_{0} t\right)
$$

where $i_{n}(t)$ is a white cyclostationary process, $i_{n 0}(t)$ is a white stationary process and $\alpha(\omega t)$ is a deterministic periodic function describing the noise amplitude modulation. We define $\alpha(\omega t)$ to be a normalized function with a maximum value of 1. This way, $\bar{i}^{2}$ no is equal to the maximum mean square noise power, $\overline{i_{n}^{2}}(t)$, which changes periodically with time. Applying the above expression for $i_{n}(t)$ to $(11), \phi(t)$ is given by

$$
\begin{aligned}
\phi(t) & =\int_{-\infty}^{t} i_{n}(\tau) \frac{\Gamma\left(\omega_{0} \tau\right)}{q_{\max }} d \tau \\
& =\int_{-\infty}^{t} i_{n 0}(\tau) \frac{\alpha\left(\omega_{0} \tau\right) \Gamma\left(\omega_{0} \tau\right)}{q_{\max }} d \tau .
\end{aligned}
$$

As can be seen, the cyclostationary noise can be treated as a stationary noise applied to a system with an effective ISF given by

$$
\Gamma_{\mathrm{eff}}(x)=\Gamma(x) \cdot \alpha(x)
$$

where $\alpha(x)$ can be derived easily from device noise characteristics and operating point. Hence, this effective ISF should be

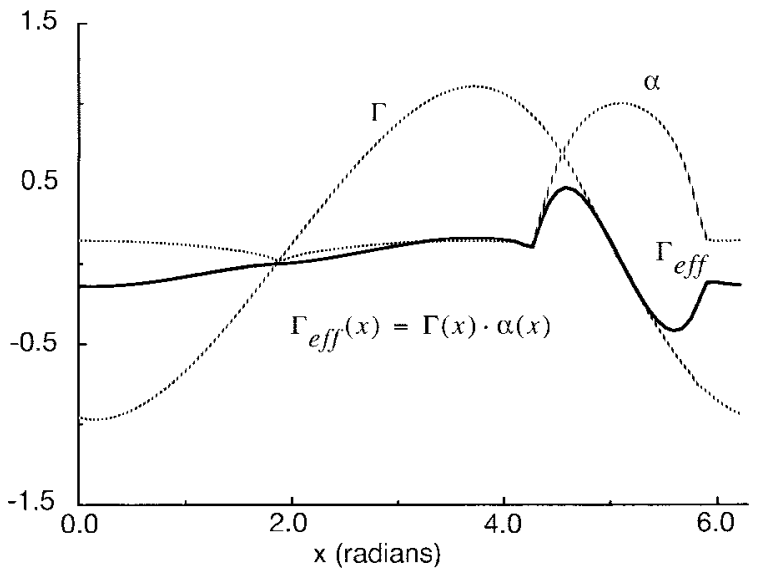

Fig. 14. $\Gamma(x), \Gamma_{\text {eff }}(x)$, and $\alpha(x)$ for the Colpitts oscillator of Fig. 5(a).

used in all subsequent calculations, in particular, calculation of the coefficients $c_{n}$.

Note that there is a strong correlation between the cyclostationary noise source and the waveform of the oscillator. The maximum of the noise power always appears at a certain point of the oscillatory waveform, thus the average of the noise may not be a good representation of the noise power.

Consider as one example the Colpitts oscillator of Fig. 5(a). The collector voltage and the collector current of the transistor are shown in Fig. 13. Note that the collector current consists of a short period of large current followed by a quiet interval. The surge of current occurs at the minimum of the voltage across the tank where the ISF is small. Functions $\Gamma(x), \alpha(x)$, and $\Gamma_{\text {eff }}(x)$ for this oscillator are shown in Fig. 14. Note that, in this case, $\Gamma_{\mathrm{eff}}(x)$ is quite different from $\Gamma(x)$, and hence the effect of cyclostationarity is very significant for the $L C$ oscillator and cannot be neglected.

The situation is different in the case of the ring oscillator of Fig. 5(b), because the devices have maximum current during the transition (when $\Gamma(x)$ is at a maximum, i.e., the sensitivity is large) at the same time the noise power is large. Functions $\Gamma(x), \alpha(x)$, and $\Gamma_{\text {eff }}(x)$ for the ring oscillator of Fig. 5(b) are shown in Fig. 15. Note that in the case of the ring oscillator $\Gamma(x)$ and $\Gamma_{\text {eff }}(x)$ are almost identical. This indicates that the cyclostationary properties of the noise are less important in the treatment of the phase noise of ring oscillators. This unfortunate coincidence is one of the reasons why ring oscillators in general have inferior phase noise performance compared to a Colpitts $L C$ oscillator. The other important reason is that ring oscillators dissipate all the stored energy during one cycle.

\section{E. Predicting Output Phase Noise with Multiple Noise Sources}

The method of analysis outlined so far has been used to predict how much phase noise is contributed by a single noise source. However, this method may be extended to multiple noise sources and multiple nodes, as individual contributions by the various noise sources may be combined by exploiting superposition. Superposition holds because the first system of Fig. 8 is linear. 


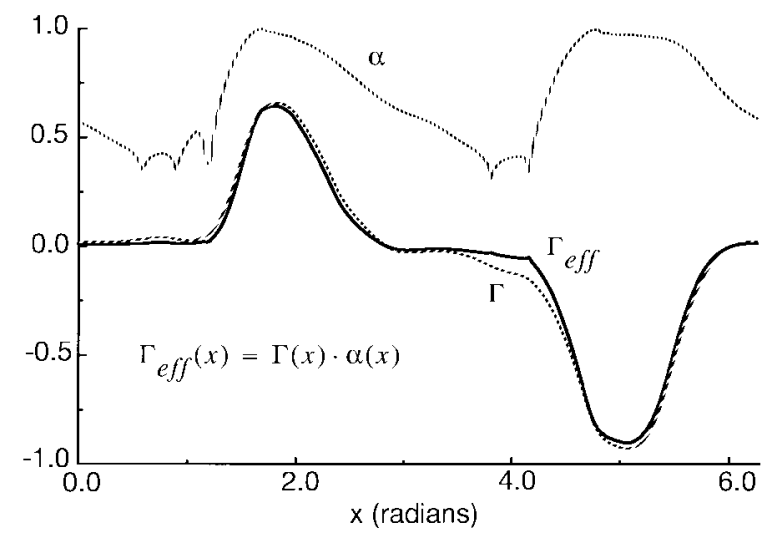

Fig. 15. $\Gamma(x), \Gamma_{\text {eff }}(x)$, and $\alpha(x)$ for the ring oscillator of Fig. 5(b).

The actual method of combining the individual contributions requires attention to any possible correlations that may exist among the noise sources. The complete method for doing so may be appreciated by noting that an oscillator has a current noise source in parallel with each capacitor and a voltage noise source in series with each inductor. The phase noise in the output of such an oscillator is calculated using the following method.

1) Find the equivalent current noise source in parallel with each capacitor and an equivalent voltage source in series with each inductor, keeping track of correlated and noncorrelated portions of the noise sources for use in later steps.

2) Find the transfer characteristic from each source to the output excess phase. This can be done as follows.

a) Find the ISF for each source, using any of the methods proposed in the Appendix, depending on the required accuracy and simplicity.

b) Find $\Gamma_{\mathrm{rms}}$ and $c_{0}$ (rms and dc values) of the ISF.

3) Use $\Gamma_{\text {rms }}$ and $c_{0}$ coefficients and the power spectrum of the input noise sources in (21) and (23) to find the phase noise power resulting from each source.

4) Sum the individual output phase noise powers for uncorrelated sources and square the sum of phase noise rms values for correlated sources to obtain the total noise power below the carrier.

Note that the amount of phase noise contributed by each noise source depends only on the value of the noise power density $\overline{i_{n}^{2}} / \Delta f$, the amount of charge swing across the effective capacitor it is injecting into $q_{\max }$, and the steady-state oscillation waveform across the noise source of interest. This observation is important since it allows us to attribute a definite contribution from every noise source to the overall phase noise. Hence, our treatment is both an analysis and design tool, enabling designers to identify the significant contributors to phase noise.

\section{F. Existing Models as Simplified Cases}

As asserted earlier, the model proposed here reduces to earlier models if the same simplifying assumptions are made.
In particular, consider the model for $L C$ oscillators in [3], as well as the more comprehensive presentation of [8]. Those models assume linear time-invariance, that all noise sources are stationary, that only the noise in the vicinity of $\omega_{0}$ is important, and that the noise-free waveform is a perfect sinusoid. These assumptions are equivalent to discarding all but the $c_{1}$ term in the ISF and setting $c_{1}=1$. As a specific example, consider the oscillator of Fig. 2. The phase noise due solely to the tank parallel resistor $R_{p}$ can be found by applying the following to (19):

$$
\begin{aligned}
\overline{i_{n}^{2}} / \Delta f & =\frac{4 k T}{R_{p}} \\
q_{\max } & =C \cdot V_{\max }
\end{aligned}
$$

where $R_{p}$ is the parallel resistor, $C$ is the tank capacitor, and $V_{\max }$ is the maximum voltage swing across the tank. Equation (19) reduces to

$$
\mathcal{L}\{\Delta \omega\}=10 \cdot \log \left[\frac{1}{2} \cdot \frac{k \mathcal{T}}{\mathcal{V}_{\max }^{2}} \cdot \frac{1}{\mathcal{R}_{p} \cdot\left(\mathcal{C} \omega_{0}\right)^{2}} \cdot\left(\frac{\omega_{0}}{\Delta \omega}\right)^{2}\right] .
$$

Since [8] assumes equal contributions from amplitude and phase portions to $\mathcal{L}_{\text {total }}\{\Delta \omega\}$, the result obtained in [8] is two times larger than the result of (29).

Assuming that the total noise contribution in a parallel tank oscillator can be modeled using an excess noise factor $F$ as in [3], (29) together with (24) result in (6). Note that the generalized approach presented here is capable of calculating the fitting parameters used in (3), $\left(F\right.$ and $\left.\Delta \omega_{1 / f}^{3}\right)$ in terms of $c_{n}$ coefficients of ISF and device $1 / f$ noise corner, $\omega_{1 / f}$.

\section{DESIGN IMPLICATIONS}

Several design implications emerge from (18), (21), and (24) that offer important insight for reduction of phase noise in the oscillators. First, they show that increasing the signal charge displacement $q_{\max }$ across the capacitor will reduce the phase noise degradation by a given noise source, as has been noted in previous works [5], [6].

In addition, the noise power around integer multiples of the oscillation frequency has a more significant effect on the closein phase noise than at other frequencies, because these noise components appear as phase noise sidebands in the vicinity of the oscillation frequency, as described by (18). Since the contributions of these noise components are scaled by the Fourier series coefficients $c_{n}$ of the ISF, the designer should seek to minimize spurious interference in the vicinity of $n \omega_{0}$ for values of $n$ such that $c_{n}$ is large.

Criteria for the reduction of phase noise in the $1 / f^{3}$ region are suggested by (24), which shows that the $1 / f^{3}$ corner of the phase noise is proportional to the square of the coefficient $c_{0}$. Recalling that $c_{0}$ is twice the dc value of the (effective) ISF function, namely

$$
c_{0}=\frac{1}{\pi} \int_{0}^{2 \pi} \Gamma_{\mathrm{eff}}(x) d x
$$

it is clear that it is desirable to minimize the dc value of the ISF. As shown in the Appendix, the value of $c_{0}$ is closely related to certain symmetry properties of the oscillation 
(a)

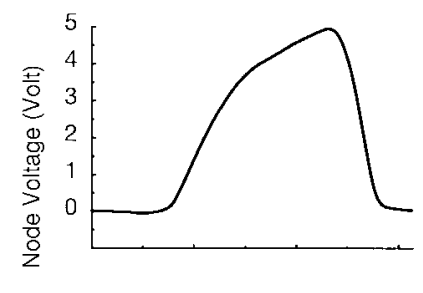

(b)

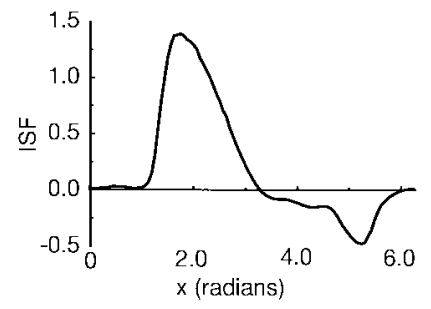

(c)

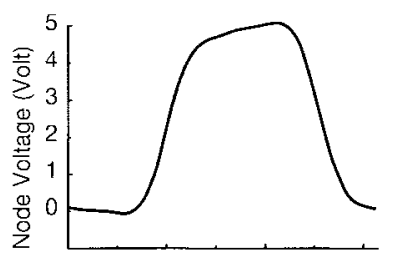

(d)

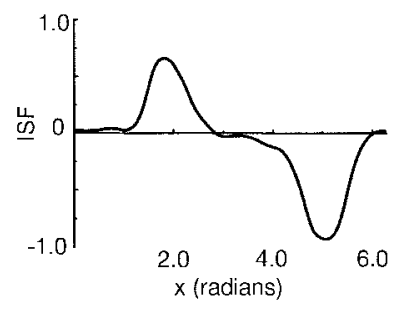

Fig. 16. (a) Waveform and (b) ISF for the asymmetrical node. (c) Waveform and (d) ISF for one of the symmetrical nodes.

waveform. One such property concerns the rise and fall times; the ISF will have a large dc value if the rise and fall times of the waveform are significantly different. A limited case of this for odd-symmetric waveforms has been observed [14]. Although odd-symmetric waveforms have small $c_{0}$ coefficients, the class of waveforms with small $c_{0}$ is not limited to odd-symmetric waveforms.

To illustrate the effect of a rise and fall time asymmetry, consider a purposeful imbalance of pull-up and pull-down rates in one of the inverters in the ring oscillator of Fig. 5(b). This is obtained by halving the channel width $W_{n}$ of the NMOS device and doubling the width $W_{p}$ of the PMOS device of one inverter in the ring. The output waveform and corresponding ISF are shown in Fig. 16(a) and (b). As can be seen, the ISF has a large dc value. For comparison, the waveform and ISF at the output of a symmetrical inverter elsewhere in the ring are shown in Fig. 16(c) and (d). From these results, it can be inferred that the close-in phase noise due to low-frequency noise sources should be smaller for the symmetrical output than for the asymmetrical one. To investigate this assertion, the results of two SPICE simulations are shown in Fig. 17. In the first simulation, a sinusoidal current source of amplitude $10 \mu \mathrm{A}$ at $f_{m}=$ $50 \mathrm{MHz}$ is applied to one of the symmetric nodes of the

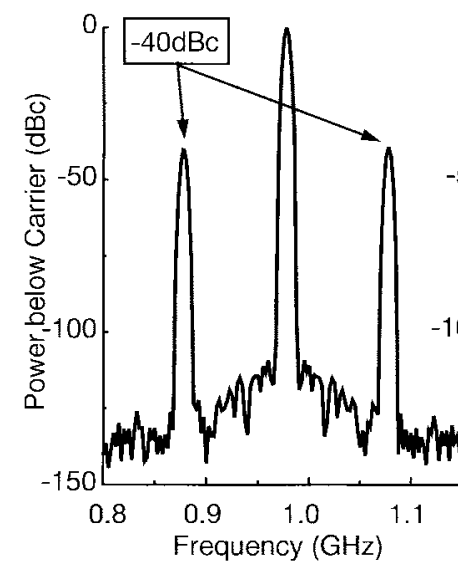

(a)

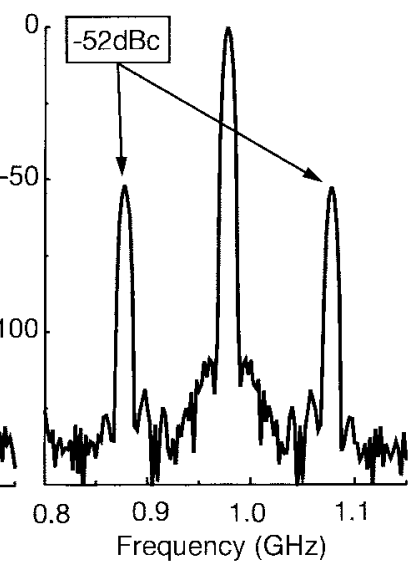

(b)
Fig. 17. Simulated power spectrum with current injection at $f_{m}=50 \mathrm{MHz}$ for (a) asymmetrical node and (b) symmetrical node.

oscillator. In the second experiment, the same source is applied to the asymmetric node. As can be seen from the power spectra of the figure, noise injected into the asymmetric node results in sidebands that are $12 \mathrm{~dB}$ larger than at the symmetric node.

Note that (30) suggests that upconversion of low frequency noise can be significantly reduced, perhaps even eliminated, by minimizing $c_{0}$, at least in principle. Since $c_{0}$ depends on the waveform, this observation implies that a proper choice of waveform may yield significant improvements in close-in phase noise. The following experiment explores this concept by changing the ratio of $W_{p}$ to $W_{n}$ over some range, while injecting $10 \mu \mathrm{A}$ of sinusoidal current at $100 \mathrm{MHz}$ into one node. The sideband power below carrier as a function of the $W_{p}$ to $W_{n}$ ratio is shown in Fig. 18. The SPICEsimulated sideband power is shown with plus symbols and the sideband power as predicted by (18) is shown by the solid line. As can be seen, close-in phase noise due to upconversion of low-frequency noise can be suppressed by an arbitrary factor, at least in principle. It is important to note, however, that the minimum does not necessarily correspond to equal transconductance ratios, since other waveform properties influence the value of $c_{0}$. In fact, the optimum $W_{p}$ to $W_{n}$ ratio in this particular example is seen to differ considerably from that used in conventional ring oscillator designs.

The importance of symmetry might lead one to conclude that differential signaling would minimize $c_{0}$. Unfortunately, while differential circuits are certainly symmetrical with respect to the desired signals, the differential symmetry disappears for the individual noise sources because they are independent of each other. Hence, it is the symmetry of each half-circuit that is important, as is demonstrated in the differential ring oscillator of Fig. 19. A sinusoidal current of $100 \mu \mathrm{A}$ at $50 \mathrm{MHz}$ injected at the drain node of one of the buffer stages results in two equal sidebands, $-46 \mathrm{~dB}$ below carrier, in the power spectrum of the differential output. Because of the voltage dependent conductance of the load devices, the individual waveform on each output node is not fully symmetrical and consequently, there will be a large 


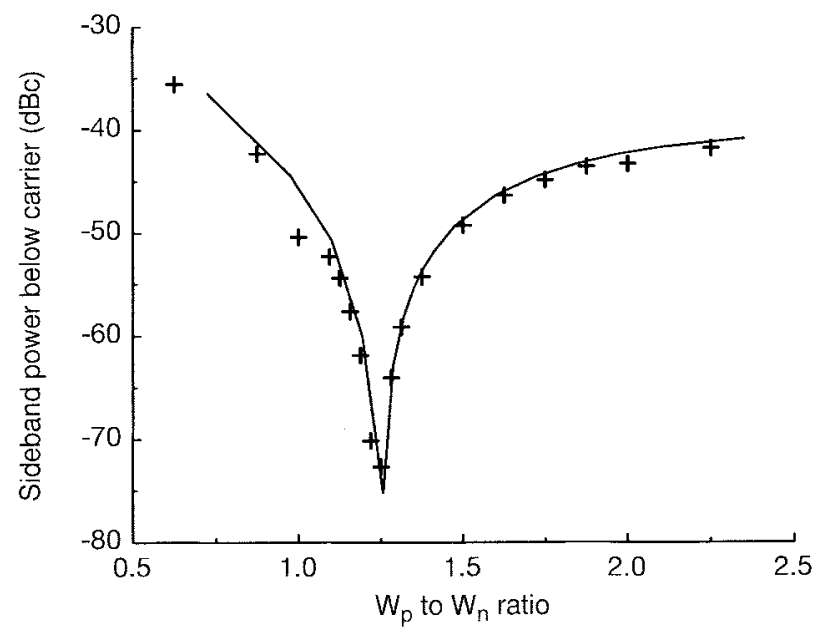

Fig. 18. Simulated and predicted sideband power for low frequency injection versus PMOS to NMOS $W / L$ ratio.
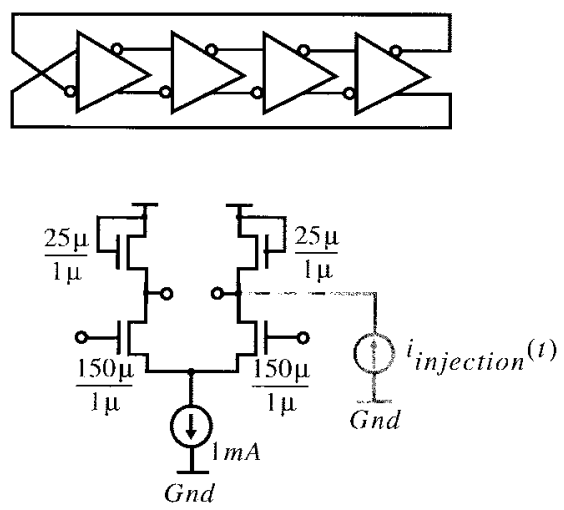

Fig. 19. Four-stage differential ring oscillator.

upconversion of noise to close-in phase noise, even though differential signaling is used.

Since the asymmetry is due to the voltage dependent conductance of the load, reduction of the upconversion might be achieved through the use of a perfectly linear resistive load, because the rising and falling behavior is governed by an $\mathrm{RC}$ time constant and makes the individual waveforms more symmetrical. It was first observed in the context of supply noise rejection [15], [16] that using more linear loads can reduce the effect of supply noise on timing jitter. Our treatment shows that it also improves low-frequency noise upconversion into phase noise.

Another symmetry-related property is duty cycle. Since the ISF is waveform-dependent, the duty cycle of a waveform is linked to the duty cycle of the ISF. Non-50\% duty cycles generally result in larger $c_{n}$ for even $n$. The high- $Q$ tank of an $L C$ oscillator is helpful in this context, since a high $Q$ will produce a more symmetric waveform and hence reduce the upconversion of low-frequency noise.

\section{EXPERIMENTAL RESULTS}

This section presents experimental verifications of the model to supplement simulation results. The first experiment ex-

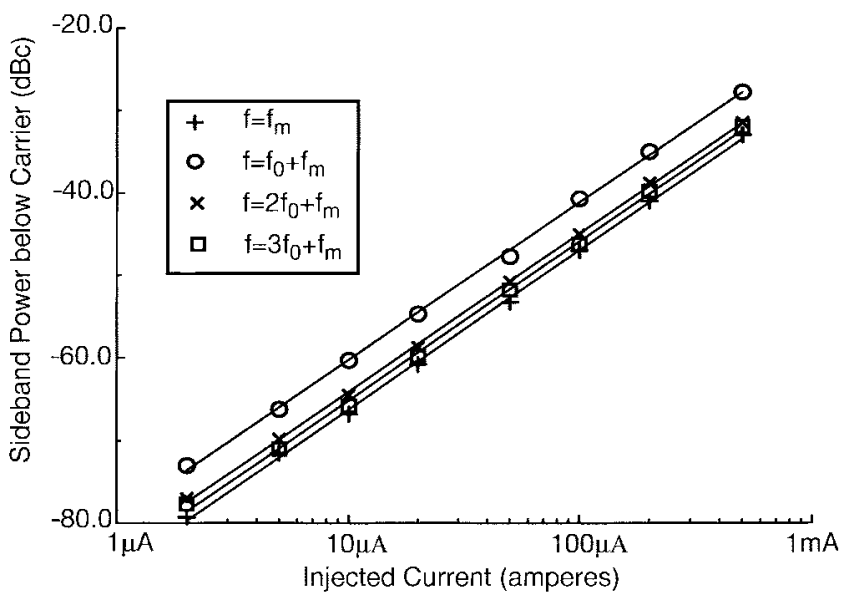

Fig. 20. Measured sideband power versus injected current at $f_{m}=100$ $\mathrm{kHz}, f_{0}+f_{m}=5.5 \mathrm{MHz}, 2 f_{0}+f_{m}=10.9 \mathrm{MHz}, 3 f_{0}+f_{m}=16.3 \mathrm{MHz}$.

amines the linearity of current-to-phase conversion using a five-stage, 5.4-MHz ring oscillator constructed with ordinary CMOS inverters. A sinusoidal current is injected at frequencies $f_{m}=100 \mathrm{kHz}, f_{0}+f_{m}=5.5 \mathrm{MHz}, 2 f_{0}+f_{m}=10.9$ $\mathrm{MHz}$, and $3 f_{0}+f_{m}=16.3 \mathrm{MHz}$, and the sideband powers at $f_{0} \pm f_{m}$ are measured as the magnitude of the injected current is varied. At any amplitude of injected current, the sidebands are equal in amplitude to within the accuracy of the measurement setup $(0.2 \mathrm{~dB})$, in complete accordance with the theory. These sideband powers are plotted versus the input injected current in Fig. 20. As can be seen, the transfer function for the input current power to the output sideband power is linear as suggested by (18). The slope of the best fit line is $19.8 \mathrm{~dB} /$ decade, which is very close to the predicted slope of $20 \mathrm{~dB} /$ decade, since excess phase $\phi(t)$ is proportional to $i(t)$, and hence the sideband power is proportional to $I^{2}$, leading to a $20-\mathrm{dB} /$ decade slope. The behavior shown in Fig. 20 verifies that the linearity of (18) holds for injected input currents orders of magnitude larger than typical noise currents.

The second experiment varies the frequency offset from an integer multiple of the oscillation frequency. An input sinusoidal current source of $20 \mu \mathrm{A}(\mathrm{rms})$ at $f_{m}, f_{0}+f_{m}$, $2 f_{0}+f_{m}$, and $3 f_{0}+f_{m}$ is applied to one node and the output is measured at another node. The sideband power is plotted versus $f_{m}$ in Fig. 21. Note that the slope in all four cases is $-20 \mathrm{~dB} /$ decade, again in complete accordance with (18).

The third experiment aims at verifying the effect of the coefficients $c_{n}$ on the sideband power. One of the predictions of the theory is that $c_{0}$ is responsible for the upconversion of low frequency noise. As mentioned before, $c_{0}$ is a strong function of waveform symmetry at the node into which the current is injected. Noise injected into a node with an asymmetric waveform (created by making one inverter asymmetric in a ring oscillator) would result in a greater increase in sideband power than injection into nodes with more symmetric waveforms. Fig. 22 shows the results of an experiment performed on a five-stage ring oscillator in which one of the stages is modified to have an extra pulldown 


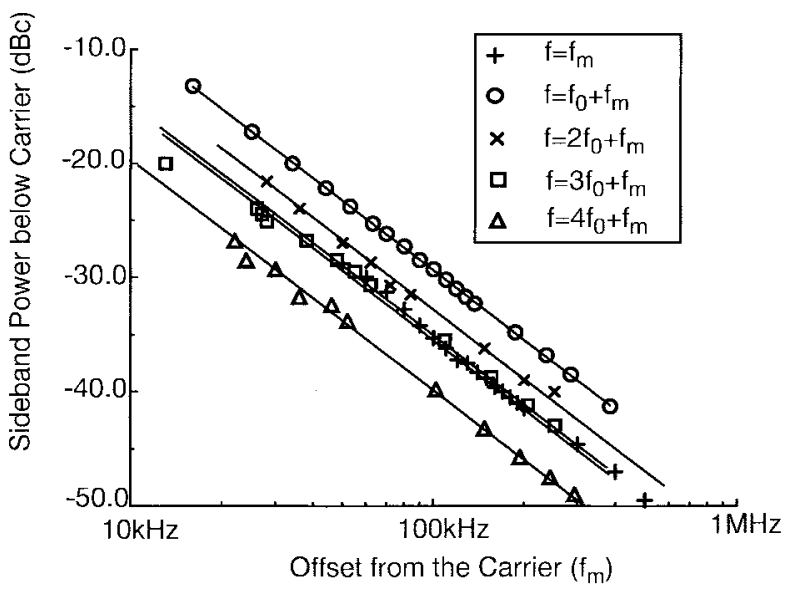

Fig. 21. Measured sideband power versus $f_{m}$, for injections in vicinity of multiples of $f_{0}$.

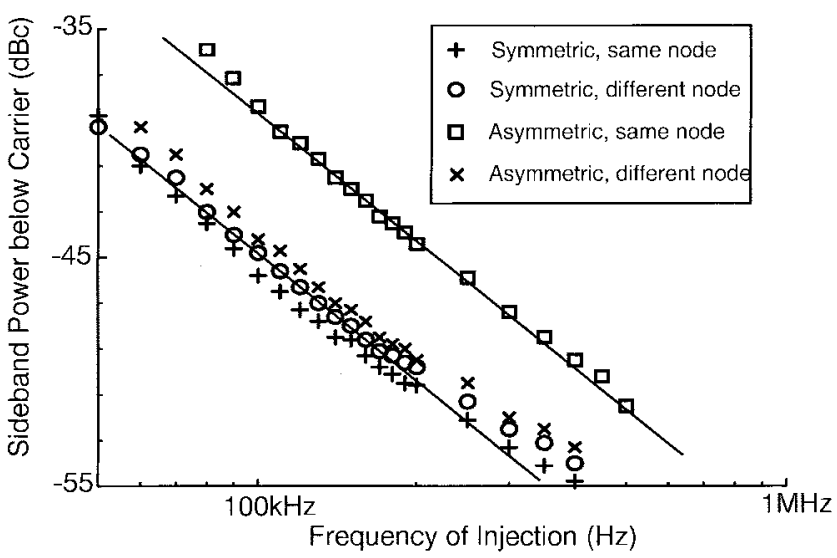

Fig. 22. Power of the sidebands caused by low frequency injection into symmetric and asymmetric nodes of the ring oscillator.

NMOS device. A current of $20 \mu \mathrm{A}$ (rms) is injected into this asymmetric node with and without the extra pulldown device. For comparison, this experiment is repeated for a symmetric node of the oscillator, before and after this modification. Note that the sideband power is $7 \mathrm{~dB}$ larger when noise is injected into the node with the asymmetrical waveform, while the sidebands due to signal injection at the symmetric nodes are essentially unchanged with the modification.

The fourth experiment compares the prediction and measurement of the phase noise for a five-stage single-ended ring oscillator implemented in a $2-\mu \mathrm{m}, 5-\mathrm{V}$ CMOS process running at $f_{0}=232 \mathrm{MHz}$. This measurement was performed using a delay-based measurement method and the result is shown in Fig. 23. Distinct $1 / f^{2}$ and $1 / f^{3}$ regions are observed. We first start with a calculation for the $1 / f^{2}$ region. For this process we have a gate oxide thickness of $t_{o x}=25 \mathrm{~nm}$ and threshold voltages of $V_{T N}=0.6 \mathrm{~V}$ and $V_{T P}=0.53 \mathrm{~V}$. All five inverters are similar with $(W / L)_{N}=3 \mu \mathrm{m} / 2 \mu \mathrm{m}$ and $(W / L)_{P}=5 \mu \mathrm{m} / 2 \mu \mathrm{m}$, and a lateral diffusion of $L_{d}=$ $0.1 \mu \mathrm{m}$. Using the process and geometry information, the total capacitance on each node, including parasitics, is calculated to be $C_{\text {total }}=35.7 \mathrm{fF}$. Therefore, $q_{\max }=C_{\text {total }} V_{\text {swing }}=$

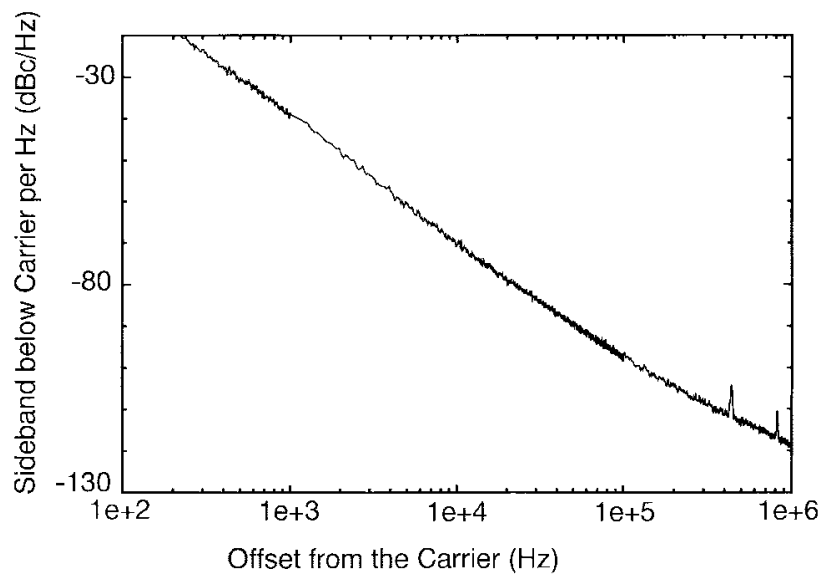

Fig. 23. Phase noise measurements for a five-stage single-ended CMOS ring oscillator. $f_{0}=232 \mathrm{MHz}, 2-\mu \mathrm{m}$ process technology.

$179 \mathrm{fC}$. As discussed in the previous section, noise current injected during a transition has the largest effect. The current noise power at this point is the sum of the current noise powers due to NMOS and PMOS devices. At this bias point, $\left(\overline{i_{n}^{2}} / \Delta f\right)_{\mathrm{NMOS}}=4 k T \gamma \mu_{n} C_{O x}\left(W / L_{\mathrm{eff}}\right)_{N}\left(V_{D D} / 2-\right.$ $\left.V_{T N}\right)=4.44 \times 10^{-24} \mathrm{~A}^{2} / \mathrm{Hz}$ and $\left(i_{n}^{2} / \Delta f\right)_{\mathrm{PMOS}}=2.19 \times$ $10^{-24} \mathrm{~A}^{2} / \mathrm{Hz}$. Using the methods outlined in the Appendix, it may be shown that $\Gamma_{\mathrm{rms}}^{2} \approx 16 / N^{3}$ for ring oscillators. Equation (21) for $N$ identical noise sources then predicts $\mathcal{L}\{\Delta f\}=10 \log \left(0.84 / \Delta f^{2}\right)$. At an offset of $\Delta f=500 \mathrm{kHz}$, this equation predicts $\mathcal{L}\{500 \mathrm{kHz}\}=-114.7 \mathrm{dBc} / \mathrm{Hz}$, in good agreement with a measurement of $-114.5 \mathrm{dBc} / \mathrm{Hz}$. To predict the phase noise in the $1 / f^{3}$ region, it is enough to calculate the $1 / f^{3}$ corner. Measurements on an isolated inverter on the same die show a $1 / f$ noise corner frequency of $250 \mathrm{kHz}$, when its input and output are shorted. The $c_{0}^{2} / 2 \Gamma_{\mathrm{rms}}^{2}$ ratio is calculated to be 0.3 , which predicts a $1 / f^{3}$ corner of $75 \mathrm{kHz}$, compared to the measured corner of $80 \mathrm{kHz}$.

The fifth experiment measures the phase noise of an 11stage ring, running at $f_{0}=115 \mathrm{MHz}$ implemented on the same die as the previous experiment. The phase noise measurements are shown in Fig. 24. For the inverters in this oscillator, $(W / L)_{N}=4 \mu \mathrm{m} / 2 \mu \mathrm{m}$ and $(W / L)_{P}=6 \mu \mathrm{m} / 2 \mu \mathrm{m}$, which results in a total capacitance of $43.5 \mathrm{fF}$ and $q_{\max }=217 \mathrm{fC}$. The phase noise is calculated in exactly the same manner as the previous experiment and is calculated to be $\mathcal{L}\{\Delta f\}=$ $10 \log \left(0.152 / \Delta f^{2}\right)$, or $-122.1 \mathrm{dBc} / \mathrm{Hz}$ at a $500-\mathrm{kHz}$ offset. The measured phase noise is $-122.5 \mathrm{dBc} / \mathrm{Hz}$, again in good agreement with predictions. The $c_{0}^{2} / 2 \Gamma_{\mathrm{rms}}^{2}$ ratio is calculated to be 0.17 which predicts a $1 / f^{3}$ corner of $43 \mathrm{kHz}$, while the measured corner is $45 \mathrm{kHz}$.

The sixth experiment investigates the effect of symmetry on $1 / f^{3}$ region behavior. It involves a seven-stage currentstarved, single-ended ring oscillator in which each inverter stage consists of an additional NMOS and PMOS device in series. The gate drives of the added transistors allow independent control of the rise and fall times. Fig. 25 shows the phase noise when the control voltages are adjusted to achieve symmetry versus when they are not. In both cases the control voltages are adjusted to keep the oscillation frequency 


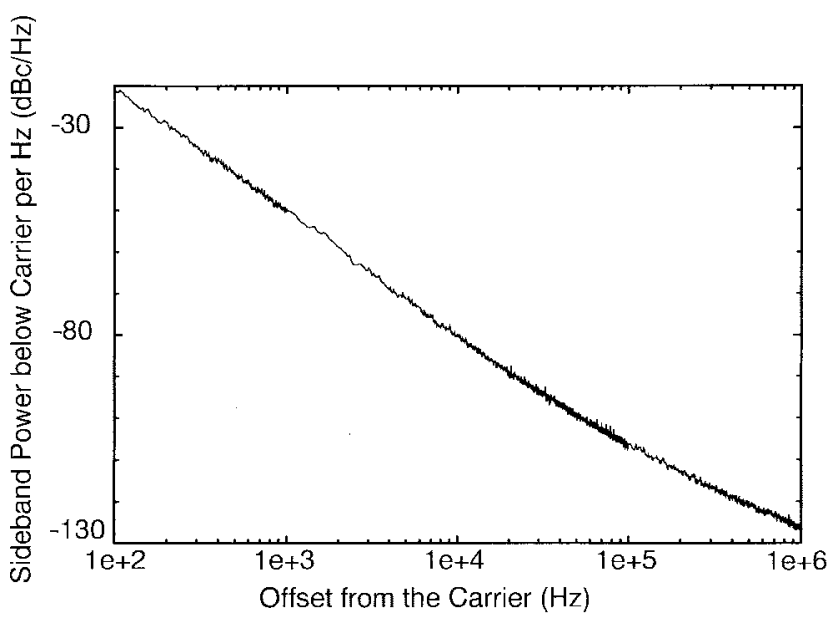

Fig. 24. Phase noise measurements for an 11-stage single-ended CMOS ring oscillator. $f_{0}=115 \mathrm{MHz}, 2-\mu \mathrm{m}$ process technology.

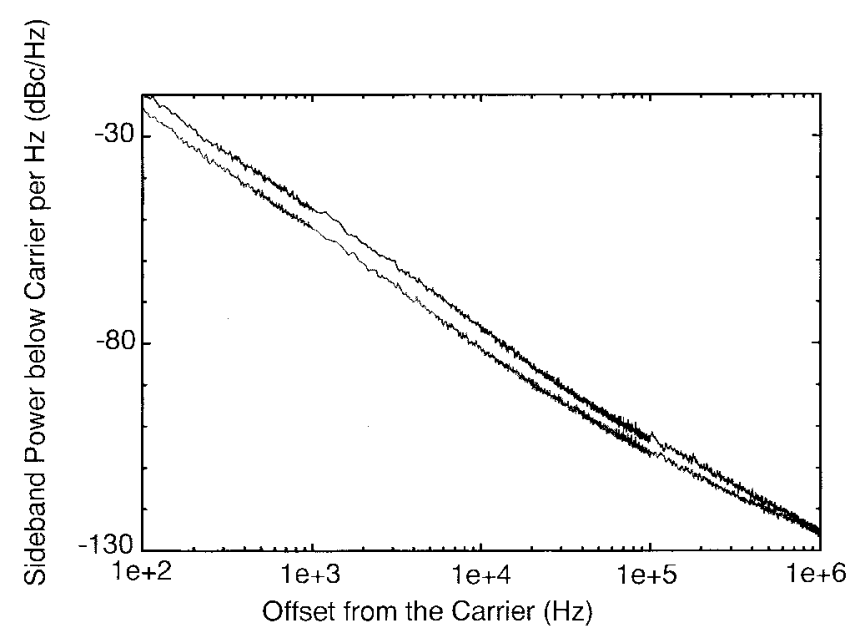

Fig. 25. Effect of symmetry in a seven-stage current-starved single-ended CMOS VCO. $f_{0}=60 \mathrm{MHz}, 2-\mu \mathrm{m}$ process technology.

constant at $60 \mathrm{MHz}$. As can be seen, making the waveform more symmetric has a large effect on the phase noise in the $1 / f^{3}$ region without significantly affecting the $1 / f^{2}$ region. Another experiment on the same circuit is shown in Fig. 26, which shows the phase noise power spectrum at a $10 \mathrm{kHz}$ offset versus the symmetry-controlling voltage. For all the data points, the control voltages are adjusted to keep the oscillation frequency at $50 \mathrm{MHz}$. As can be seen, the phase noise reaches a minimum by adjusting the symmetry properties of the waveform. This reduction is limited by the phase noise in $1 / f^{2}$ region and the mismatch in transistors in different stages, which are controlled by the same control voltages.

The seventh experiment is performed on a four-stage differential ring oscillator, with PMOS loads and NMOS differential stages, implemented in a $0.5-\mu \mathrm{m}$ CMOS process. Each stage is tapped with an equal-sized buffer. The tail current source has a quiescent current of $108 \mu \mathrm{A}$. The total capacitance on each of the differential nodes is calculated to be $C_{\text {total }}=49 \mathrm{fF}$ and the voltage swing is $V_{\text {swing }}=1.2 \mathrm{~V}$, which results in $q_{\max }=58.8 \mathrm{fF}$. The total channel noise current on each node

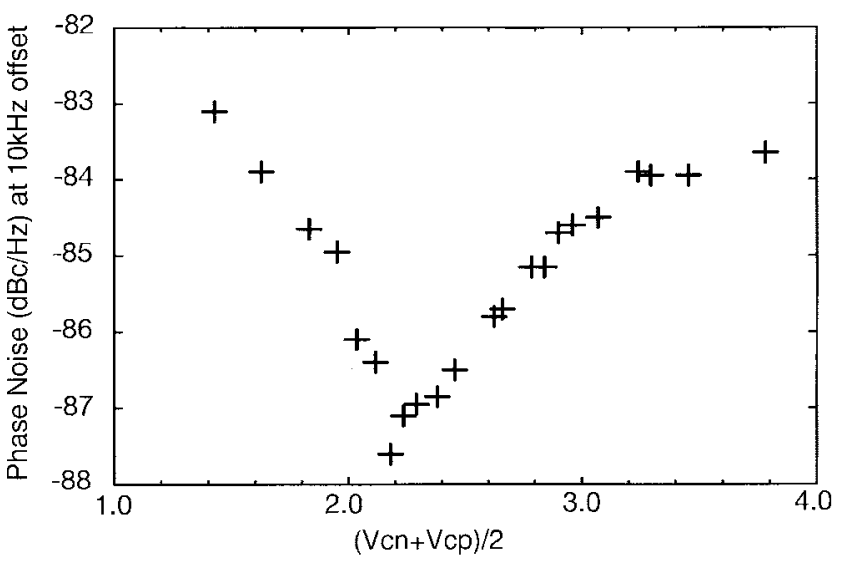

Fig. 26. Sideband power versus the voltage controlling the symmetry of the waveform. Seven-stage current-starved single-ended CMOS VCO. $f_{0}=50$ $\mathrm{MHz}, 2-\mu \mathrm{m}$ process technology.

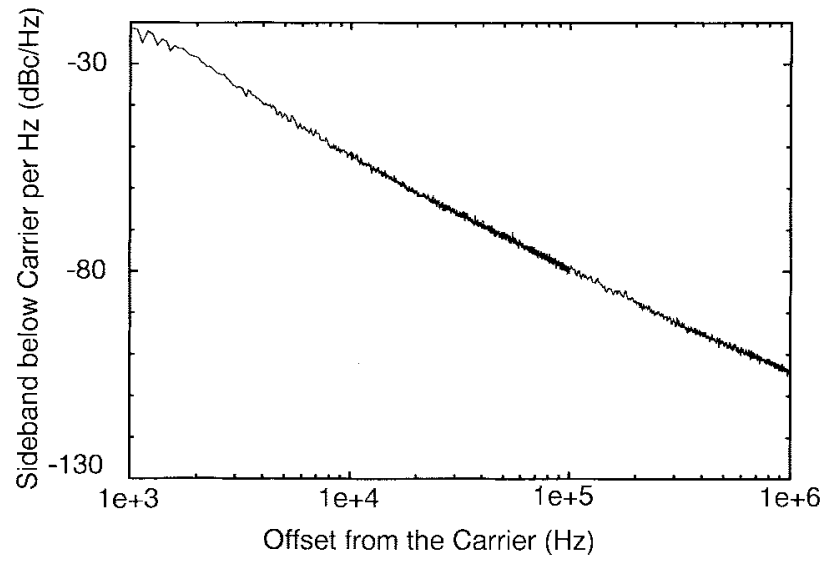

Fig. 27. Phase noise measurements for a four-stage differential CMOS ring oscillator. $f_{0}=200 \mathrm{MHz}, 0.5-\mu \mathrm{m}$ process technology.

is $\left(\overline{i_{n}^{2}} / \Delta f\right)_{\text {total }}=2.63 \times 10^{-23} \mathrm{~A}^{2} / \mathrm{Hz}$. Using these numbers for $N=4$, the phase noise in the $1 / f^{2}$ region is predicted to be $\mathcal{L}\{\Delta f\}=10 \log \left(48.1 / \Delta f^{2}\right)$, or $-103.2 \mathrm{dBc} / \mathrm{Hz}$ at an offset of $1 \mathrm{MHz}$, while the measurement in Fig. 27 shows a phase noise of $-103.9 \mathrm{dBc} / \mathrm{Hz}$, again in agreement with prediction. Also note that despite differential symmetry, there is a distinct $1 / f^{3}$ region in the phase noise spectrum, because each half circuit is not symmetrical.

The eighth experiment investigates cyclostationary effects in the bipolar Colpitts oscillator of Fig. 5(a), where the conduction angle is varied by changing the capacitive divider ratio $n=C_{1} /\left(C_{1}+C_{2}\right)$ while keeping the effective parallel capacitance $C_{\mathrm{eq}}=C_{1} C_{2} /\left(C_{1}+C_{2}\right)$ constant to maintain an $f_{0}$ of $100 \mathrm{MHz}$. As can be seen in Fig. 28, increasing $n$ decreases the conduction angle, and thereby reduces the effective $\Gamma_{\text {rms }}$, leading to an initial decrease in phase noise. However, the oscillation amplitude is approximately given by $V_{\text {tank }}=2 R_{\operatorname{tank}} I_{E E}(1-n)$, and therefore decreases for large values of $n$. The phase noise ultimately increases for large $n$ as a consequence. There is thus a definite value of $n$ (here, about 0.2 ) that minimizes the phase noise. This result provides a theoretical basis for the common rule-of-thumb that one should 


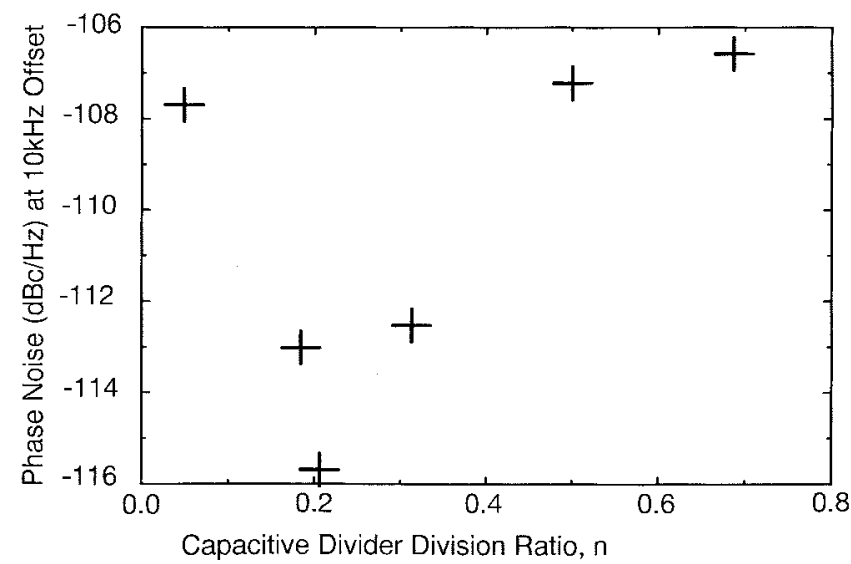

Fig. 28. Sideband power versus capacitive division ratio. Bipolar $L C$ Colpitts oscillator $f_{0}=100 \mathrm{MHz}$.

use $C_{2} / C_{1}$ ratios of about four (corresponding to $n=0.2$ ) in Colpitts oscillators [17].

\section{CONCLUSION}

This paper has presented a model for phase noise which explains quantitatively the mechanism by which noise sources of all types convert to phase noise. The power of the model derives from its explicit recognition of practical oscillators as time-varying systems. Characterizing an oscillator with the ISF allows a complete description of the noise sensitivity of an oscillator and also allows a natural accommodation of cyclostationary noise sources.

This approach shows that noise located near integer multiples of the oscillation frequency contributes to the total phase noise. The model specifies the contribution of those noise components in terms of waveform properties and circuit parameters, and therefore provides important design insight by identifying and quantifying the major sources of phase noise degradation. In particular, it shows that symmetry properties of the oscillator waveform have a significant effect on the upconversion of low frequency noise and, hence, the $1 / f^{3}$ corner of the phase noise can be significantly lower than the $1 / f$ device noise corner. This observation is particularly important for MOS devices, whose inferior $1 / f$ noise has been thought to preclude their use in high-performance oscillators.

\section{APPENDIX}

\section{CAlculation of the IMPUlse Sensitivity Function}

In this Appendix we present three different methods to calculate the ISF. The first method is based on direct measurement of the impulse response and calculating $\Gamma(x)$ from it. The second method is based on an analytical state-space approach to find the excess phase change caused by an impulse of current from the oscillation waveforms. The third method is an easy-to-use approximate method.

\section{A. Direct Measurement of Impulse Response}

In this method, an impulse is injected at different relative phases of the oscillation waveform and the oscillator simulated

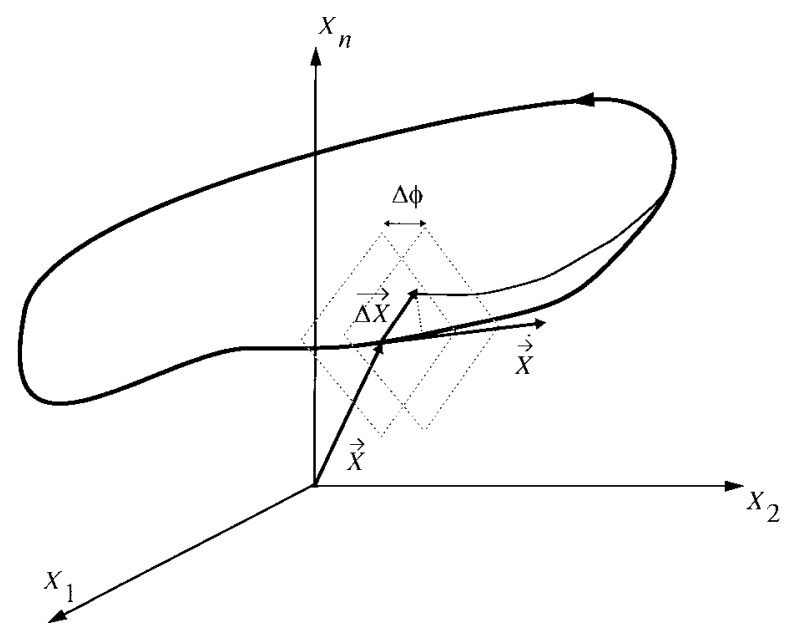

Fig. 29. State-space trajectory of an $n$ th-order oscillator.

for a few cycles afterwards. By sweeping the impulse injection time across one cycle of the waveform and measuring the resulting time shift $\Delta t, h_{\phi}(t, \tau)$ can calculated noting that $\Delta \phi=2 \pi \Delta t / T$, where $T$ is the period of oscillation. Fortunately, many implementations of SPICE have an internal feature to perform the sweep automatically. Since for each impulse one needs to simulate the oscillator for only a few cycles, the simulation executes rapidly. Once $h_{\phi}(t, \tau)$ is found, the ISF is calculated by multiplication with $q_{\max }$. This method is the most accurate of the three methods presented.

\section{B. Closed-Form Formula for the ISF}

An $n$ th-order system can be represented by its trajectory in an $n$-dimensional state-space. In the case of a stable oscillator, the state of the system, represented by the state vector, $\vec{X}$, periodically traverses a closed trajectory, as shown in Fig. 29. Note that the oscillator does not necessarily traverse the limit cycle with a constant velocity.

In the most general case, the effect of a group of external impulses can be viewed as a perturbation vector $\Delta \vec{X}$ which suddenly changes the state of the system to $\vec{X}+\Delta \vec{X}$. As discussed earlier, amplitude variations eventually die away, but phase variations do not. Application of the perturbation impulse causes a certain change in phase in either a negative or positive direction, depending on the state-vector and the direction of the perturbation. To calculate the equivalent time shift, we first find the projection of the perturbation vector on a unity vector in the direction of motion, i.e., the normalized velocity vector

$$
l=\Delta \vec{X} \cdot \frac{\dot{\vec{X}}}{|\dot{\vec{X}}|}
$$

where $l$ is the equivalent displacement along the trajectory, and $\dot{\vec{X}}$ is the first derivative of the state vector. Note the scalar nature of $l$, which arises from the projection operation. The equivalent time shift is given by the displacement divided by 
the "speed" $|\dot{\bar{X}}|$

$$
\Delta t=\frac{l}{|\dot{\vec{X}}|}=\Delta \vec{X} \cdot \frac{\dot{\vec{X}}}{|\dot{\vec{X}}|^{2}}
$$

which results in the following equation for excess phase caused by the perturbation:

$$
\Delta \phi=2 \pi \frac{\Delta t}{T}=\frac{2 \pi}{T}\left(\Delta \bar{X} \cdot \frac{\dot{\vec{X}}}{|\dot{\bar{X}}|^{2}}\right) .
$$

In the specific case where the state variables are node voltages, and an impulse is applied to the $i$ th node, there will be a change in $\Delta V_{i}$ given by (10). Equation (33) then reduces to

$$
\Delta \phi_{i}=\frac{2 \pi}{T} \cdot \frac{\Delta q_{i}}{C_{i}} \cdot \frac{\dot{v}_{i}}{|\dot{\vec{v}}|^{2}}
$$

where $|\dot{\vec{v}}|^{2}$ is the norm of the first derivative of the waveform vector and $\dot{v}_{i}$ is the derivative of the $i$ th node voltage. Equation (34), together with the normalized waveform function $f$ defined in (1), result in the following:

$$
\Delta \phi=\frac{\Delta q}{q_{i}} \cdot \frac{f_{i}^{\prime}}{\left|\overrightarrow{f^{\prime}}\right|^{2}}
$$

where $f_{i}^{\prime}$ represents the derivative of the normalized waveform on node $i$, hence

$$
\Gamma_{i}(x)=\frac{f_{i}^{\prime}}{\left|\overrightarrow{f^{\prime}}\right|^{2}}=\frac{f_{i}^{\prime}}{\sum_{j=1}^{n} f_{j}^{\prime 2}} .
$$

It can be seen that this expression for the ISF is maximum during transitions (i.e., when the derivative of the waveform function $f$ is maximum), and this maximum value is inversely proportional to the maximum derivative. Hence, waveforms with larger slope show a smaller peak in the ISF function.

In the special case of a second-order system, one can use the normalized waveform $f$ and its derivative as the state variables, resulting in the following expression for the ISF:

$$
\Gamma(x)=\frac{f^{\prime}}{f^{\prime 2}+f^{\prime \prime 2}}
$$

where $f^{\prime \prime}$ represents the second derivative of the function $f$. In the case of an ideal sinusoidal oscillator $f=\cos (x)$, so that $\Gamma(\omega t)=-\sin (\omega t)$, which is consistent with the argument of Section III. This method has the attribute that it computes the ISF from the waveform directly, so that simulation over only one cycle of $f$ is required to obtain all of the necessary information.

\section{Calculation of ISF Based on the First Derivative}

This method is actually a simplified version of the second approach. In certain cases, the denominator of (36) shows little variation, and can be approximated by a constant. In such a case, the ISF is simply proportional to the derivative of the waveform. A specific example is a ring oscillator with $N$
Calculation of Impulse Sensitivity Function

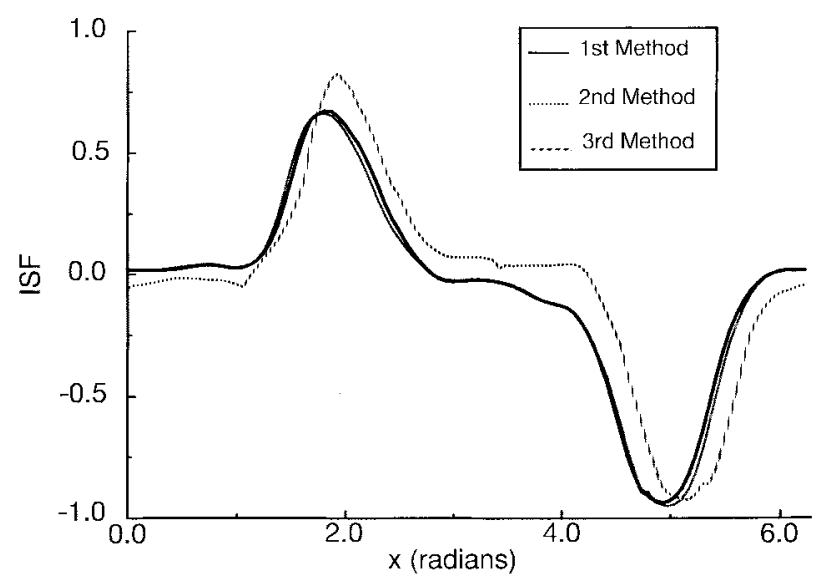

Fig. 30. ISF's obtained from different methods.

identical stages. The denominator may then be approximated by $f_{\max }^{\prime 2}$

$$
\Gamma_{i}(x)=\frac{f_{i}^{\prime}(x)}{f_{\max }^{\prime 2}} .
$$

Fig. 30 shows the results obtained from this method compared with the more accurate results obtained from methods $A$ and $B$. Although this method is approximate, it is the easiest to use and allows a designer to rapidly develop important insights into the behavior of an oscillator.

\section{ACKNOWLEDGMENT}

The authors would like to thank T. Ahrens, R. Betancourt, R. Farjad-Rad, M. Heshami, S. Mohan, H. Rategh, H. Samavati, D. Shaeffer, A. Shahani, K. Yu, and M. Zargari of Stanford University and Prof. B. Razavi of UCLA for helpful discussions. The authors would also like to thank M. Zargari, R. Betancourt, B. Amruturand, J. Leung, J. Shott, and Stanford Nanofabrication Facility for providing several test chips. They are also grateful to Rockwell Semiconductor for providing access to their phase noise measurement system.

\section{REFERENCES}

[1] E. J. Baghdady, R. N. Lincoln, and B. D. Nelin, "Short-term frequency stability: Characterization, theory, and measurement," Proc. IEEE, vol. 53, pp. 704-722, July 1965.

[2] L. S. Cutler and C. L. Searle, "Some aspects of the theory and measurement of frequency fluctuations in frequency standards," Proc. IEEE, vol. 54, pp. 136-154, Feb. 1966.

[3] D. B. Leeson, "A simple model of feedback oscillator noises spectrum," Proc. IEEE, vol. 54, pp. 329-330, Feb. 1966.

[4] J. Rutman, "Characterization of phase and frequency instabilities in precision frequency sources; Fifteen years of progress," Proc. IEEE, vol. 66, pp. 1048-1174, Sept. 1978.

[5] A. A. Abidi and R. G. Meyer, "Noise in relaxation oscillators," IEEE J. Solid-State Circuits, vol. SC-18, pp. 794-802, Dec. 1983.

[6] T. C. Weigandt, B. Kim, and P. R. Gray, "Analysis of timing jitter in CMOS ring oscillators," in Proc. ISCAS, June 1994, vol. 4, pp. 27-30.

[7] J. McNeil, "Jitter in ring oscillators," in Proc. ISCAS, June 1994, vol. 6, pp. 201-204.

[8] J. Craninckx and M. Steyaert, "Low-noise voltage controlled oscillators using enhanced LC-tanks," IEEE Trans. Circuits Syst.-II, vol. 42, pp. 794-904, Dec. 1995. 
[9] B. Razavi, "A study of phase noise in CMOS oscillators," IEEE $J$. Solid-State Circuits, vol. 31, pp. 331-343, Mar. 1996.

[10] B. van der Pol, "The nonlinear theory of electric oscillations," Proc. IRE, vol. 22, pp. 1051-1086, Sept. 1934.

[11] N. Minorsky, Nonlinear Oscillations. Princeton, NJ: Van Nostrand, 1962.

[12] P. A. Cook, Nonlinear Dynamical Systems. New York: Prentice Hall, 1994.

[13] W. A. Gardner, Cyclostationarity in Communications and Signal Processing. New York: IEEE Press, 1993.

[14] H. B. Chen, A. van der Ziel, and K. Amberiadis, "Oscillator with oddsymmetrical characteristics eliminates low-frequency noise sidebands," IEEE Trans. Circuits Syst., vol. CAS-31, Sept. 1984.

[15] J. G. Maneatis, "Precise delay generation using coupled oscillators," IEEE J. Solid-State Circuits, vol. 28, pp. 1273-1282, Dec. 1993.

[16] C. K. Yang, R. Farjad-Rad, and M. Horowitz, "A 0.6mm CMOS 4Gb/s transceiver with data recovery using oversampling," in Symp. VLSI Circuits, Dig. Tech. Papers, June 1997.

[17] D. DeMaw, Practical RF Design Manual. Englewood Cliffs, NJ: Prentice-Hall, 1982, p. 46.

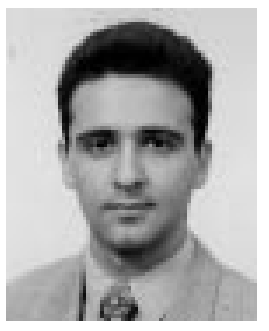

Ali Hajimiri (S'95) was born in Mashad, Iran, in 1972. He received the B.S. degree in electronics engineering from Sharif University of Technology in 1994 and the M.S. degree in electrical engineering from Stanford University, Stanford, CA, in 1996, where he is currently engaged in research toward the Ph.D. degree in electrical engineering.

He worked as a Design Engineer for Philips on a BiCMOS chipset for the GSM cellular units from 1993 to 1994. During the summer of 1995, he worked for Sun Microsystems, Sunnyvale, CA, on the UltraSparc microprocessor's cache RAM design methodology. Over the summer of 1997, he worked at Lucent Technologies (Bell-Labs), where he investigated low phase noise integrated oscillators. He holds one European and two U.S. patents.

Mr. Hajimiri is the Bronze medal winner of the 21st International Physics Olympiad, Groningen, Netherlands.

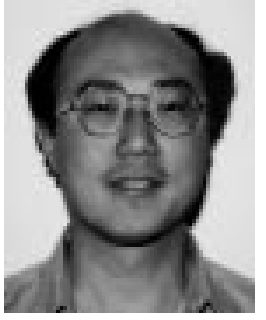

Thomas H. Lee (M'83) received the S.B., S.M., Sc.D. degrees from the Massachusetts Institute of Technology (MIT), Cambridge, in 1983, 1985, and 1990, respectively.

He worked for Analog Devices Semiconductor, Wilmington, MA, until 1992, where he designed high-speed clock-recovery PLL's that exhibit zero jitter peaking. He then worked for Rambus Inc., Mountain View, CA, where he designed the phaseand delay-locked loops for $500 \mathrm{MB} / \mathrm{s}$ DRAM's. In 1994, he joined the faculty of Stanford University, Stanford, CA, as an Assistant Professor, where he is primarily engaged in research into microwave applications for silicon IC technology, with a focus on CMOS IC's for wireless communications.

Dr. Lee was recently named a recipient of a Packard Foundation Fellowship award and is the author of The Design of CMOS Radio-Frequence Integrated Circuits (Cambridge University Press). He has twice received the "Best Paper" award at ISSCC. 